\title{
PROPERTIES OF THE OPEN CLUSTER TOMBAUGH 1 FROM HIGH-RESOLUTION SPECTROSCOPY AND
} uvbyCaH $\beta$ PHOTOMETRY*

\author{
João V. Sales Silva ${ }^{1,2}$, Giovanni Carraro ${ }^{1,6}$, Barbara J. Anthony-Twarog ${ }^{3}$, Christian Moni Bidin ${ }^{4}$, Edgardo Costa ${ }^{5}$, and \\ BRuCE A. Twarog ${ }^{3}$ \\ ${ }^{1}$ ESO, Alonso de Cordova 3107, 19001, Santiago de Chile, Chile; joaovictor@on.br, gcarraro@eso.org \\ ${ }^{2}$ Observatório Nacional/MCT, Rua Gen. José Cristino, 77, 20921-400, Rio de Janeiro, Brazil \\ ${ }^{3}$ Department of Physics and Astronomy, University of Kansas, Lawrence, KS 66045-7582, USA; bjat,btwarog@ku.edu \\ ${ }^{4}$ Instituto de Astronomia, Universidad Católica del Norte, Av. Angamos 0610, Casilla 1280, Antofagasta, Chile; cmoni@ucn.cl \\ ${ }^{5}$ Departamento de Astronomia, Universidad de Chile, Casilla 36-D, Santiago, Chile; costa@ das.uchile.cl \\ Received 2015 September 9; accepted 2015 October 20; published 2015 December 21
}

\begin{abstract}
Open clusters can be the key to deepening our knowledge on various issues involving the structure and evolution of the Galactic disk and details of stellar evolution because a cluster's properties are applicable to all its members. However, the number of open clusters with detailed analysis from high-resolution spectroscopy or precision photometry imposes severe limitations on studies of these objects. To expand the number of open clusters with well-defined chemical abundances and fundamental parameters, we investigate the poorly studied, anticenter open cluster Tombaugh 1 . Using precision $u v b y \mathrm{CaH} \beta$ photometry and high-resolution spectroscopy, we derive the cluster's reddening, obtain photometric metallicity estimates, and, for the first time, present a detailed abundance analysis of 10 potential cluster stars (nine clump stars and one Cepheid). Using the radial position from the cluster center and multiple color indices, we have isolated a sample of unevolved, probable single-star members of Tombaugh 1. From 51 stars, the cluster reddening is found to be $E(b-y)=0.221 \pm 0.006$ or $E(B-V)=0.303 \pm 0.008$, where the errors refer to the internal standard errors of the mean. The weighted photometric metallicity from $m_{1}$ and $h k$ is $[\mathrm{Fe} / \mathrm{H}]=-0.10 \pm 0.02$, while a match to the Victoria-Regina Strömgren isochrones leads to an age of $0.95 \pm 0.10 \mathrm{Gyr}$ and an apparent modulus of $(m-M)=13.10 \pm 0.10$. Radial velocities identify six giants as probable cluster members, and the elemental abundances of $\mathrm{Fe}, \mathrm{Na}, \mathrm{Mg}, \mathrm{Al}$, $\mathrm{Si}, \mathrm{Ca}, \mathrm{Ti}, \mathrm{Cr}, \mathrm{Ni}, \mathrm{Y}, \mathrm{Ba}, \mathrm{Ce}$, and $\mathrm{Nd}$ have been derived for both the cluster and the field stars. Tombaugh 1 appears to be a typical inner thin disk, intermediate-age open cluster of slightly subsolar metallicity, located just beyond the solar circle, with solar elemental abundance ratios except for the heavy s-process elements, which are a factor of two above solar. Its metallicity is consistent with a steep metallicity gradient in the galactocentric region between 9.5 and $12 \mathrm{kpc}$. Our study also shows that Cepheid XZ CMa is not a member of Tombaugh 1 and reveals that this Cepheid presents signs of barium enrichment, making it a probable binary star.
\end{abstract}

Key words: open clusters and associations: general - open clusters and associations: individual (Tombaugh 1) stars: abundances - stars: atmospheres

\section{INTRODUCTION}

Tombaugh 1 is a star cluster in the Canis Majoris constellation at $\alpha=07^{\mathrm{h}} 00^{\mathrm{m}} 29^{\mathrm{s}}, \delta=-20^{\circ} 34^{\prime} 00^{\prime \prime}, \quad$ and $\ell$, $b=232^{\circ} .22,-7^{\circ} .32$ (2000.0 equinox), discovered in 1938 by Clyde Tombaugh (Tombaugh 1938). A quick glance at any sky image shows only a small enhancement of stars in a rich Galactic field, a primary reason why Tombaugh 1 has been studied very little until now. As detailed in Section 2, estimates of its fundamental parameters vary strongly from author to author, photometric data are scanty, and spectroscopic data are totally absent.

There are, however, several reasons to consider this cluster particularly interesting. With a Galactic latitude of -7.32 and an assumed a distance of 2-3 kpc (Carraro \& Patat 1995; Piatti et al. 2004), Tombaugh 1 is situated $\sim 300-400$ pc below the Galactic plane, a location rare among open clusters of the presumed age of Tombaugh 1 ( $\lesssim 10^{9}$ years). The direction to Tombaugh 1 intersects the Perseus and Orion arms in the third Galactic quadrant, and the cluster was for some time associated

\footnotetext{
* Based on observations carried out at Las Campanas Observatory (program ID: CN2009B-042) and Cerro Tololo Inter-American Observatory.

6 On leave from Dipartimento di Fisica e Astronomia, Universitá di Padova, Italy.
}

with the putative Canis Major dwarf galaxy (Bellazzini et al. 2004). Although the discussion of this topic has been dormant for some time (Carraro et al. 2008), it would still be valuable to determine the cluster distance with enough precision to associate it with either the Perseus or, possibly, the Orion arm extension in the third Galactic quadrant (Vásquez et al. 2008). Moreover, a distance of 2-3 kpc places Tombaugh 1 at a galactocentric distance of $10-11 \mathrm{kpc}$, a location where some observations indicate an abrupt change in the abundance gradient of the disk (see, e.g., Twarog et al. 1997; Lépine et al. 2011). Improved cluster distance and metallicity estimates would be of paramount importance in probing this picture of the chemical properties of the outer Galactic disk. Metallicity, in particular, has never been measured reliably, only inferred either from photometric indices or by comparison with isochrones (Piatti et al. 2004).

With these goals in mind, we present new Strömgren $u v b y \mathrm{CaH} \beta$ photometry for a large field around the cluster and high-resolution spectroscopy of 10 potential cluster stars (nine red giant clump stars and one Cepheid). This unique data set will be used to provide precise estimates of the cluster basic parameters, in particular reddening, distance, age, and overall and elemental metallicity. 
The paper is organized as follows. Section 2 gives details on the limited investigation of the cluster to date. In Section 3 we present the photometric and spectroscopic observations and their reduction, as well as a description of the radial-velocity determinations for membership. Section 4 details the derivation of the cluster properties from photometry, and Section 5 is devoted to the spectroscopic abundance analysis. In Section 6 we discuss the results of our spectroscopic analysis in detail. Section 7 interprets Tombaugh 1 in the context of galactic evolution and summarizes our findings.

\section{TOMBAUGH 1: BACKGROUND}

Tombaugh discovered Tombaugh 1 and Tombaugh 2 during the trans-Neptunian planet search at Lowell Observatory (Tombaugh 1938, 1941), reporting an apparent diameter for Tombaugh 1 of $\sim 5^{\prime}$, with typical cluster stars having a visual magnitude of 14-15. His short description of the cluster notes that the field in the direction of Tombaugh 1 is extremely rich, suggesting that this might be why the cluster went undetected for so long.

As discussed in Turner (1983), Haffner (1957) and Tifft (1959) independently rediscovered Tombaugh 1, reporting discrepant values for the cluster decl.; the Haffner (1957) position is incorrect. Tifft (1959) noted the cluster because the Cepheid XZ CMa lies about one cluster diameter northward of the cluster center. Turner (1983) provided the first estimates for the cluster fundamental parameters from an analysis of $U B V$ photoelectric photometry of 26 stars, including five likely members and 10 possible members selected by radial location in the cluster and position in the two-color diagram, the $U B V$ diagram also indicating $E(B-V)=0.27 \pm 0.01 \mathrm{mag}$. Turner (1983) measured a cluster diameter of $\sim 10^{\prime}$ and estimated a distance and age of $1.26 \mathrm{kpc}((m-M)=11.34 \pm 0.04)$ and $\sim 800 \mathrm{Myr}$, respectively, using the Hyades cluster adjusted to solar metallicity as a reference, though the sparse photometry extended barely $1.5 \mathrm{mag}$ below the top of the turnoff. Lastly, Turner (1983) suggested that Tombaugh 1 hosts a probable blue straggler star (BSS), later confirmed by Ahumada \& Lapasset (1995).

Turner (1983) also investigated the membership of Cepheid XZ CMa with Tombaugh 1. Turner suggested that XZ CMa is not a member of Tombaugh 1 . However, this conclusion was never subjected to a more rigorous analysis based on highresolution spectroscopy. So, we also spectroscopically analyzed XZ CMa to confirm or exclude the cluster membership. In fact, cluster Cepheids are important to fixing the distance scale (see, e.g., Majaess et al. 2013a, 2013b).

The first CCD study of Tombaugh 1, limited to VI, was carried out by Carraro \& Patat (1995), covering an area $6^{\prime}$ on a side, essentially the cluster core (Turner 1983). Very different values for some of the cluster basic parameters were found: reddening, distance, and age of $E(B-V)=0.40 \pm 0.05$, $3 \mathrm{kpc}((m-M)=13.60 \pm 0.2)$, and $1 \mathrm{Gyr}$, respectively, tied to color-magnitude diagram (CMD) matches to theoretical isochrones. While this study dealt with only two filters and field star contamination makes it difficult to clearly identify the cluster turnoff, the parameter differences are not unexpected given the sparse sample of the earlier study.

Piatti et al. (2004) presented a more extensive CCD study using Washington photometry, covering a large area around the cluster. The cluster was found to be $1.3 \mathrm{Gyr}$ old from a combination of CMD morphology and isochrone fits, assuming
$[\mathrm{Fe} / \mathrm{H}]=-0.40$, with distance and reddening estimates intermediate between the Turner (1983) and Carraro \& Patat (1995) values. An attempt was also made to directly measure the metallicity using Washington photometry, obtaining [Fe/ $\mathrm{H}]=-0.30$ with a large uncertainty of $\pm 0.25 \mathrm{dex}$.

Finally, on the basis of stellar data from PPMXL ${ }^{7}$ and the Two Micron All Sky Survey (2MASS), Kharchenko et al. (2013) obtained some spatial, structural, kinematic, and astrophysical parameters of Tombaugh 1. In particular, they determined for Tombaugh 1 an age of $1.16 \mathrm{Gyr}$, a reddening $E(B-V)=0.281$, and a distance of 2642 pc $((m-M)=12.98)$, values similar to those obtained by Piatti et al. (2004). Kharchenko et al. (2013) also estimated the

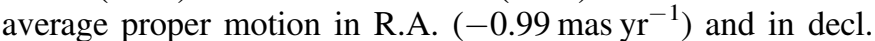
(3.97 $\mathrm{mas} \mathrm{yr}^{-1}$ ), but they did not estimate the average radial and galactic space velocities of Tombaugh 1 .

\section{OBSERVATIONS AND DATA REDUCTION}

\subsection{Photometry}

Photometry for Tombaugh 1 was secured in 2010 December during a five-night run using the Cerro Tololo Inter-American Observatory $1.0 \mathrm{~m}$ telescope operated by the SMARTS consortium. ${ }^{8}$ The telescope is equipped with an STA $4064 \times 4064$ CCD camera ${ }^{9}$ with $15 \mu \mathrm{m}$ pixels, yielding a scale of 0 !' $289 / \mathrm{pixel}$ and a field of view (FOV) of $20^{\prime} \times 20^{\prime}$ at the Cassegrain focus of the telescope.

In Table 1 we present the log of our Strömgren observations, together with exposure times and air masses. A total of 75 images were acquired for Tombaugh 1 . All observations were carried out under photometric conditions with good seeing (0.8-1.2 arcsec). A sample image of the covered field is shown in Figure 1.

Basic calibration of the CCD frames was done using the Yale/SMARTS $y 4 k$ reduction script based on the IRAF $^{10}$ package CCDRED, and the photometry was performed using IRAF's DAOPHOT and PHOTCAL packages. Instrumental magnitudes were extracted following the point-spread function (PSF) method (Stetson 1987) using a quadratic, spatially variable master PSF (the PENNY function). Finally, the PSF photometry was aperture-corrected using corrections determined from aperture photometry of bright, isolated stars in the field.

Standard stars for the extended Strömgren system were observed on one of the photometric nights on which Tombaugh 1 was observed. We additionally employed observations of secondary standard fields in several open clusters, using the same telescope and instrument one year later, to derive the form of the calibration equations. The clusters observed in 2011 December were M67 (Nissen et al. 1987), NGC 2287 (Schmidt 1984), and NGC 2516 (Snowden 1975). The zero point for each calibration equation applied to Tombaugh 1 was anchored by observations of eight field star standards obtained on 2010 December 7. Standard values were obtained from the catalogs of Olsen $(1983,1993,1994)$ for $u v b y$, from Hauck \& Mermilliod (1998) for $\mathrm{H} \beta$ values, and from Twarog \&

\footnotetext{
PPMXL is a catalog of positions, proper motions, 2MASS, and optical photometry of 900 million stars and galaxies. For more information, see http:// vo.uni-hd.de/ppmxl

8 http://www.astro.yale.edu/smarts

9 http://www.astronomy.ohio-state.edu/Y4KCam/detector

10 IRAF is distributed by the National Optical Astronomy Observatory, operated by the Association of Universities for Research in Astronomy, Inc., under cooperative agreement with the National Science Foundation.
} 
Table 1

Strömgren Photometric Observations of Tombaugh 1

\begin{tabular}{|c|c|c|c|c|}
\hline Date & Field & Filter & Exposures (s) & $\begin{array}{c}\text { Airmass } \\
\text { (X) }\end{array}$ \\
\hline 2010 Dec 05 & Tombaugh 1 & $\begin{array}{c}y \\
b \\
\mathrm{H} \beta_{\text {wide }} \\
\mathrm{Ca} \\
\mathrm{H} \beta_{\text {narrow }}\end{array}$ & $\begin{array}{c}60,600 \\
60,600 \\
60,600 \\
120,1200 \\
120,1200\end{array}$ & $\begin{array}{c}1.03 \\
1.02 \\
1.01-1.02 \\
1.01 \\
1.02\end{array}$ \\
\hline 2010 Dec 06 & Tombaugh 1 & $\begin{array}{c}y \\
b \\
v \\
\mathrm{Ca}\end{array}$ & $\begin{array}{c}2 \times 60,900 \\
2 \times 60,900 \\
60,900 \\
120,1500\end{array}$ & $\begin{array}{c}1.03 \\
1.02 \\
1.01-1.03 \\
1.04-1.08\end{array}$ \\
\hline 2010 Dec 07 & Tombaugh 1 & $\begin{array}{c}u \\
b \\
v \\
\mathrm{Ca} \\
\mathrm{H} \beta_{\text {wide }} \\
\mathrm{H} \beta_{\text {narrow }}\end{array}$ & $\begin{array}{c}10,300 \\
10,60 \\
10,100 \\
10,200 \\
10,60 \\
10,200\end{array}$ & $\begin{array}{c}1.02 \\
1.01 \\
1.01 \\
1.01 \\
1.01-1.01 \\
1.02\end{array}$ \\
\hline 2010 Dec 08 & Tombaugh 1 & $\begin{array}{c}u \\
v \\
\mathrm{H} \beta_{\text {narrow }}\end{array}$ & $\begin{array}{c}2 \times 20,2002000 \\
20,90,900 \\
20,150,1500\end{array}$ & $\begin{array}{c}1.02-1.03 \\
1.01-1.03 \\
1.05\end{array}$ \\
\hline 2010 Dec 09 & Tombaugh 1 & $\begin{array}{c}y \\
b \\
v \\
\mathrm{Ca} \\
\mathrm{H} \beta_{\text {wide }} \\
\mathrm{H} \beta_{\text {narrow }}\end{array}$ & $\begin{array}{c}10,60,120,600 \\
60,180,900 \\
100,200,1200 \\
100,300,1800 \\
100,200,1200 \\
10,300,1800\end{array}$ & $\begin{array}{l}1.46-1.51 \\
1.35-1.39 \\
1.25-1.28 \\
1.04-1.05 \\
1.16-1.18 \\
1.09-1.11\end{array}$ \\
\hline 2010 Dec 09 & Tombaugh 1 & $\begin{array}{c}y \\
b \\
v \\
\mathrm{Ca} \\
\mathrm{H} \beta_{\text {wide }} \\
\mathrm{H} \beta_{\text {narrow }} \\
u\end{array}$ & $\begin{array}{c}60,600 \\
180,900 \\
200,900 \\
300,1500 \\
200,900 \\
300,1500 \\
400,1800\end{array}$ & $\begin{array}{c}1.47-1.49 \\
1.38-1.40 \\
1.30 \\
1.07-1.08 \\
1.30 \\
1.13-1.14 \\
1.03-1.04\end{array}$ \\
\hline
\end{tabular}

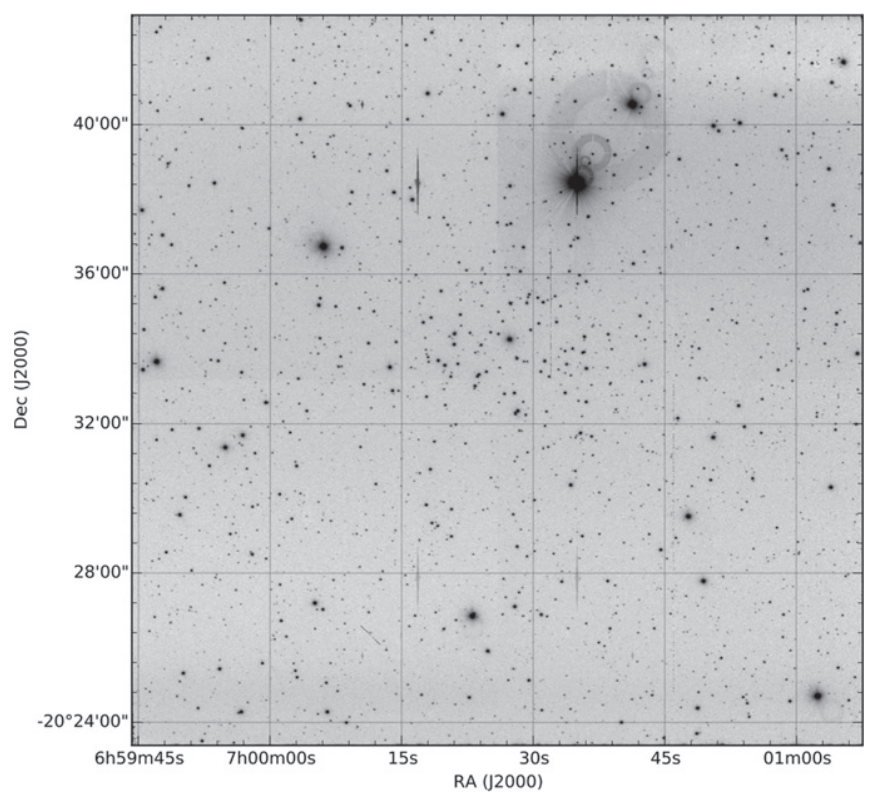

Figure 1. A $1200 \mathrm{~s}$ exposure in the $v$ filter. North is down and east to the right. The field is $20^{\prime}$ on a side.
Table 2

Calibration Equations Summary

\begin{tabular}{lccc}
\hline \hline Index & Slope & Color Term & Std. Deviation \\
\hline$V$ & 1.00 & 0.05 & 0.010 \\
$b-y$ & 1.01 & $\ldots$ & 0.002 \\
$h k$ & 1.07 & $\ldots$ & 0.009 \\
$\mathrm{H} \beta$ & 1.18 & $\ldots$ & 0.015 \\
$m_{1}$ & 0.92 & -0.075 & 0.025 \\
$c_{1}$ & 1.06 & $\ldots$ & 0.021 \\
\hline
\end{tabular}

Anthony-Twarog (1995) for $h k$ index values for the field star standards.

Table 2 summarizes the calibration equations' slopes and color terms. Following a standard practice for Strömgren photometry, calibration of $(b-y)$ would require that separate slopes be determined for cooler dwarfs as distinct from warmer dwarfs and giants. Insufficient cool dwarf standards were observed to accomplish this. The slope listed in Table 2 is appropriate for giants and dwarfs with $(b-y)_{0} \leqslant 0.42$; application of this slope to dwarfs redder than this is an unavoidable extrapolation. Bluer dwarfs represent the only class for which $m_{1}$ and $c_{1}$ calibrations could be established with any confidence. The errors listed in Table 2 represent the standard deviation of the calibrated values about the standard values for the field star standards, indicating the external precision of the zero points of the calibration equations. The final calibrated catalog was then cross-correlated with 2MASS to convert pixel (i.e., detector) coordinates into R.A. and decl. for J2000.0 equinox, thus providing 2MASS-based astrometry. An excerpt of the optical photometric table used in this investigation is illustrated in Table 3 . Figure 2 shows the trend of errors with $V$ magnitude. The $V$ and $b-y$ data remain below 0.02 mag uncertainty to the limit of Table $3(V=18.5)$, while errors in the remaining indices begin to rise above this value at $V=16.5,17.25,17.25$, and 17.5 for $c_{1}, m_{1}, h k$, and $\mathrm{H} \beta$, respectively.

\subsection{Spectroscopy and Radial Velocities}

Over the night of 2010 January 5, we observed 10 potential cluster stars (nine clump stars and one Cepheid; see Section 4.1) with the Inamori-Magellan Areal Camera \& Spectrograph (IMACS; Dressler et al. 2006) attached to the Magellan telescope $(6.5 \mathrm{~m})$ located at Las Campanas, Chile. The spectra were obtained using the Multi-Object Echelle (MOE) mode with two exposures, one of $900 \mathrm{~s}$ and the other of $1200 \mathrm{~s}$. Our spectra have a resolution of $R \sim 20,000$, while the spectral coverage depends on the location of the star on the multislit mask, but it generally goes from 4200 to $9100 \AA$. The detector consists of a mosaic with eight CCDs with gaps of about $0.93 \mathrm{~mm}$ between the CCDs, causing small gaps in stellar spectra.

The spectra were reduced following the standard procedures using IRAF, which includes CCD bias and flat-fielding correction, spectrum extraction, wavelength calibration, and sky subtraction using the tasks CCDPROC, DOECSLIT, ECIDENTIFY, and BACKGROUND, respectively. For each CCD, we performed bias and flat-fielding correction separately, after which we used the IRAF tasks IMCREATE and IMCOPY to join the CCDs and create the mosaic. The cosmic rays were removed with the IRAF Laplacian edge-detection routine (van Dokkum 2001), and the radial velocities (RV) were obtained from the 
Table 3

Excerpt of the Photometric Catalog of Tombaugh 1

\begin{tabular}{|c|c|c|c|c|c|c|c|c|c|c|c|c|c|}
\hline$\alpha(2000)$ & $\delta(2000)$ & $V$ & $b-y$ & $h k$ & $\mathrm{H} \beta$ & $m_{1}$ & $c_{1}$ & $\sigma_{V}$ & $\sigma_{b y}$ & $\sigma_{m 1}$ & $\sigma_{c 1}$ & $\sigma_{h k}$ & $\sigma_{\beta}$ \\
\hline 105.1726 & -20.6754 & 7.179 & 0.826 & 1.377 & 2.532 & 0.453 & 0.187 & 0.0195 & 0.0204 & 0.0205 & 0.0067 & 0.0213 & 0.0074 \\
\hline 105.0252 & -20.6121 & 9.426 & 0.232 & 0.326 & 2.769 & 0.119 & 0.793 & 0.0021 & 0.0030 & 0.0037 & 0.0035 & 0.0040 & 0.0026 \\
\hline 105.2605 & -20.4113 & 10.227 & 0.044 & 0.234 & 2.915 & 0.145 & 1.012 & 0.0023 & 0.0034 & 0.0042 & 0.0039 & 0.0046 & 0.0026 \\
\hline 105.1471 & -20.6414 & 10.326 & 0.323 & 0.550 & 2.673 & 0.158 & 0.406 & 0.0023 & 0.0039 & 0.0055 & 0.0057 & 0.0058 & 0.0042 \\
\hline 105.0961 & -20.4473 & 10.400 & 0.268 & 0.473 & 2.716 & 0.209 & 0.613 & 0.0018 & 0.0026 & 0.0034 & 0.0034 & 0.0037 & 0.0027 \\
\hline 105.2796 & -20.5640 & 10.463 & 1.232 & 1.976 & 2.574 & 0.661 & -0.319 & 0.0016 & 0.0023 & 0.0038 & 0.0076 & 0.0037 & 0.0022 \\
\hline 104.9458 & -20.5607 & 10.502 & 0.410 & 0.585 & 2.582 & 0.167 & 0.251 & 0.0016 & 0.0023 & 0.0030 & 0.0030 & 0.0032 & 0.0019 \\
\hline 105.0569 & -20.5582 & 10.641 & 1.387 & 1.216 & 2.754 & 0.050 & 0.134 & 0.0017 & 0.0024 & 0.0035 & 0.0056 & 0.0035 & 0.0020 \\
\hline 105.0750 & -20.6804 & 10.733 & 1.084 & 1.836 & 2.550 & 0.639 & -0.147 & 0.0017 & 0.0023 & 0.0033 & 0.0046 & 0.0034 & 0.0019 \\
\hline 105.1990 & -20.4917 & 10.947 & 0.379 & 0.568 & 2.620 & 0.134 & 0.411 & 0.0016 & 0.0024 & 0.0032 & 0.0035 & 0.0034 & 0.0020 \\
\hline 105.0211 & -20.4531 & 11.051 & 0.797 & 1.338 & 2.550 & 0.471 & 0.199 & 0.0017 & 0.0023 & 0.0032 & 0.0037 & 0.0033 & 0.0020 \\
\hline
\end{tabular}

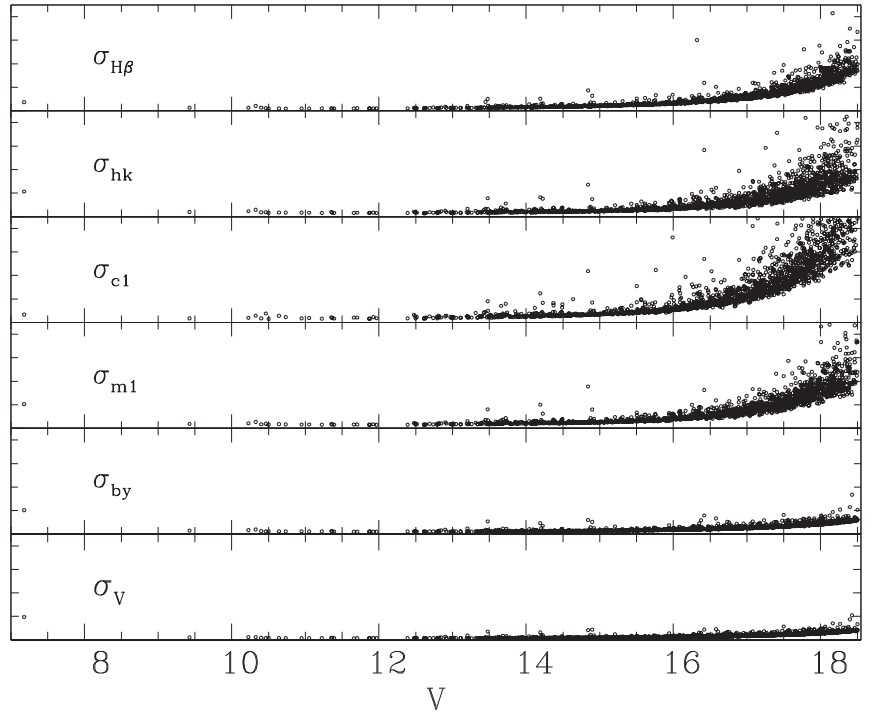

Figure 2. Trend of global photometric errors in magnitude and colors as a function of $V$ magnitude. See text for details.

wavelength shift of the unblended absorption lines of $\mathrm{Fe}$ covering the entire wavelength range. The values of wavelength shift were measured by a line-by-line comparison between observed and laboratory wavelengths, with the line's center of observed wavelength being determined through the task SPLOT in IRAF. To derived the final RV, we applied a zeropoint offset correction using the task FXCOR in IRAF to crosscorrelate the telluric lines of the observed spectra with the telluric lines of the high-resolution FEROS solar spectrum collected by us in a previous run (Moni Bidin et al. 2012). To calculate the heliocentric velocities and combine the spectra of different exposures, we used the IRAF tasks RVCORRECT and SCOMBINE, respectively. We took the star's heliocentric radial velocity to be the average of the two epochs measured and the error to be the difference between the two values multiplied by 0.63 (small-sample statistics; see Keeping 1962). The nominal signal-to-noise ratio $(\mathrm{S} / \mathrm{N})$ was evaluated by measuring with IRAF the rms flux fluctuation in selected continuum windows. The values at $6000 \AA$ are shown in Table 4 .

Table 4 gives some information about the observed stars: IDs (Carraro \& Patat 1995); R.A., decl., $V$, and $b-y$ from Table 3 and $V-I$ photometry (Carraro \& Patat 1995); heliocentric $\mathrm{RV}\left(\mathrm{RV}_{1}\right.$ and $\left.\mathrm{RV}_{2}\right)$ at two epochs and their mean values $(\langle\mathrm{RV}\rangle)$; projected rotational velocities $(v \sin i)$; and the spectral signal-to-noise ratio at $6000 \AA$. We estimated the projected rotational velocities, $v \sin i$, by a spectral synthesis technique using unblended $\mathrm{Fe}$ lines analyzed with model atmospheres, a macroturbulent velocity of $3 \mathrm{~km} \mathrm{~s}^{-1}$, and limb darkening and instrumental broadening corresponding to IMACS spectral resolution. For some stars with low $v \sin i$, it was possible to determine only an upper limit because of the insensitivity of the spectral synthesis to $v \sin i$ below $2.7 \mathrm{~km} \mathrm{~s}^{-1}$.

In the literature, there is no information about the radial velocity of Tombaugh 1 . So, to determine the membership of stars, we first found a group of stars with similar heliocentric $\mathrm{RV}$ in the sample to have a preliminary cluster radial velocity and a list of members that could be iteratively refined. The stars with RV within $2 \sigma$ of the cluster mean heliocentric radial velocity were classified as members of Tombaugh 1 . The membership of the Cepheid XZ CMa (star 806) was not defined by its heliocentric radial velocity because its RV is variable due to pulsations. So, we classified $\mathrm{XZ} \mathrm{CMa} \mathrm{as} \mathrm{a} \mathrm{nonmember} \mathrm{of}$ Tombaugh 1 because its metallicity $([\mathrm{Fe} / \mathrm{H}]=-0.53)$ is much lower than the metallicity of stars classified as members of Tombaugh 1 (see Table 9). We identify six red clump giants belonging to Tombaugh 1 and derive a mean cluster heliocentric radial velocity of $81.1 \pm 2.5 \mathrm{~km} \mathrm{~s}^{-1}$.

\section{CLUSTER PARAMETERS FROM PHOTOMETRY}

As discussed previously, one of the primary challenges in identifying and studying Tombaugh 1 is isolating the moderately populated cluster from the rich stellar background. This is particularly important for defining directly the fundamental cluster parameters of reddening and metallicity and indirectly the distance and age. The challenge is illustrated in Figure 3, where the $V, b-y$ CMD for the entire field of study is presented. Red giants observed as part of this investigation and found to be probable radial-velocity members are plotted as red starred points; probable nonmembers are open red triangles. The complexity of the stellar population mix in this region of the galaxy is evident and will be discussed in detail in a future paper. To enhance our definition of the cluster, we first reinvestigate the radial profile of the cluster.

\subsection{Star Counts and Cluster Size}

To quantify the amount of field-star contamination, we performed star counts to derive an estimate of the cluster center and size. Using an array of positions covering the field of the CCD, we derived a density contour map and calculated the density inside each grid step by a kernel estimate (Carraro et al. 
Table 4

Potential Cluster Stars Observed with IMACS in the Field of Tombaugh 1

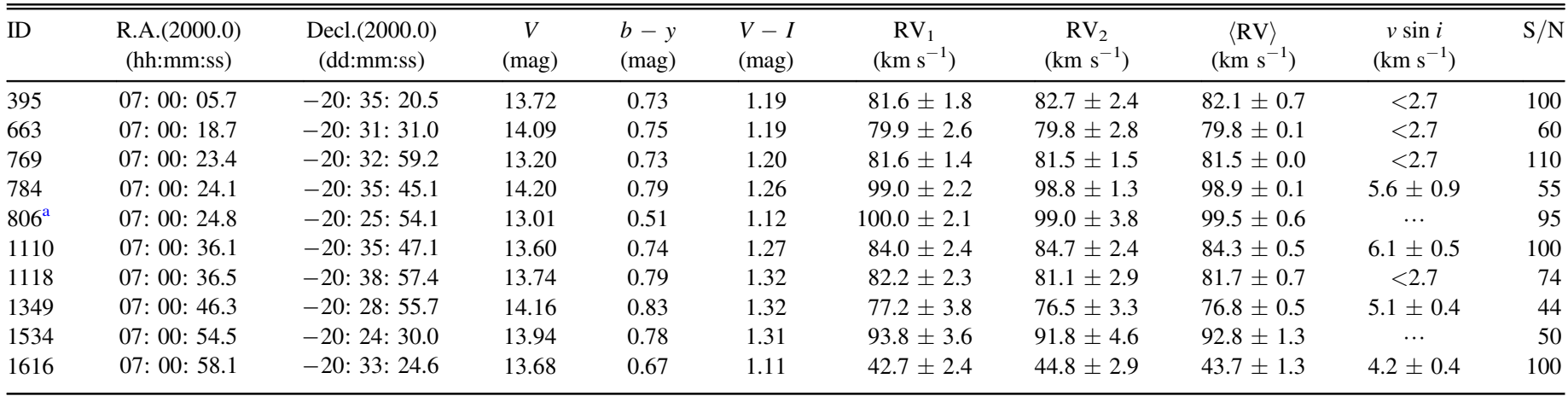

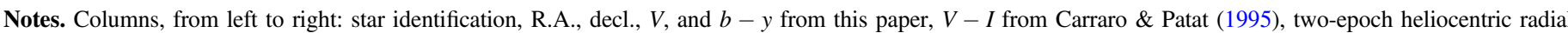
velocities $\left(\mathrm{RV}_{1}\right.$ and $\left.\mathrm{RV}_{2}\right)$ and their mean values $(\langle\mathrm{RV}\rangle)$, projected rotational velocities $(v \sin i)$, and the spectral signal-to-noise ratio at $6000 \AA$.

a The classical Cepheid XZ CMa.

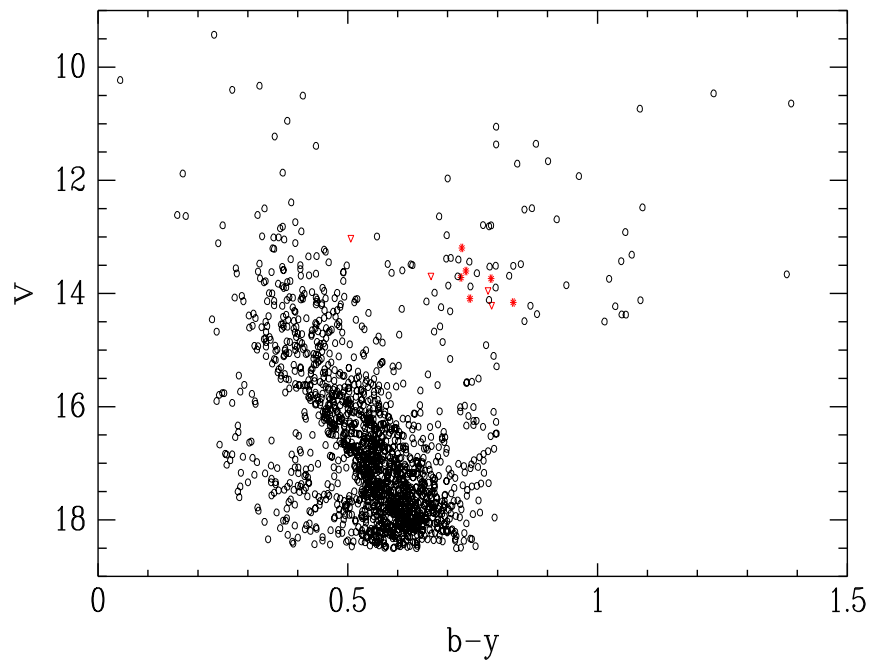

Figure 3. Color-magnitude diagram of Tombaugh 1. Red symbols identify the 10 potential cluster stars observed with IMACS. Starred points are probable members, and open triangles are nonmembers. See text for details.

2014a). This is shown in Figure 4, which confirms the appearance of Figure 1 in which Tombaugh 1 is far from being a symmetric object. The cluster looks elongated in the direction northeast to southwest, and the highest peak does not represent the center of a uniform star distribution. The largest peak is located at R.A. $=105^{\circ} .11$, decl. $=-20^{\circ} .58$, while the nominal center of the cluster is clearly displaced to the northeast direction at R.A. $=105^{\circ} .13$, decl. $=-20.54$. The loose and irregular shape of Tombaugh 1 may be the result of its dynamical evolution that is due to its tidal interaction with the Milky Way. However, little kinematic information beyond the cluster radial velocity exists to confirm this scenario. Highquality proper motions could go a long way toward defining the direction of the cluster motion and test if this coincides with the direction of the apparent cluster deformation, indicating if Tombaugh 1 has indeed been tidally disturbed.

To isolate probable cluster members, assumed to be those stars that lie within the cluster boundaries, we derive the cluster radial surface-density profile shown in Figure 5. This has been computed by drawing concentric rings centered on the nominal cluster center. This is motivated by the fact that, while the densest central regions look distorted, the cluster halo still

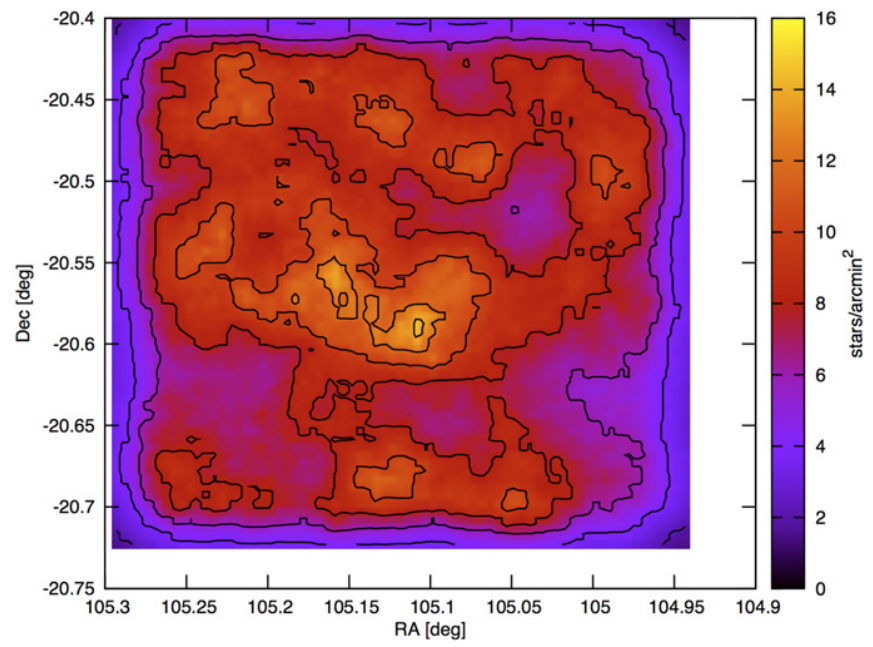

Figure 4. Density contour map for Tombaugh 1 field. North is up, east to the left, and the field corresponds to $20^{\prime} \times 20^{\prime}$ on the sky.

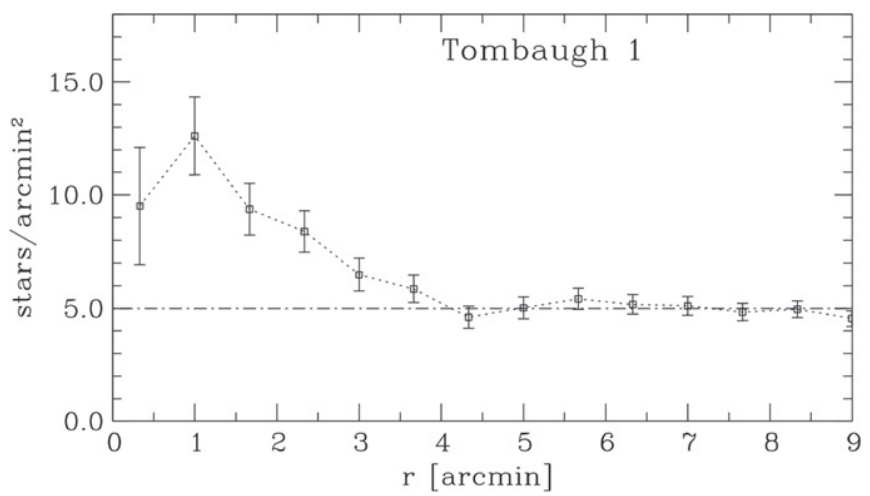

Figure 5. Radial surface-density profile. To define concentric rings, we used the nominal cluster center.

retains a more spherical profile. Star counts level off at $\sim 4^{\prime}$ from the cluster nominal center, close to the value reported by Turner (1983). The mean density in the field surrounding the cluster is 5 stars $\operatorname{arcmin}^{-2}$ (see also Figure 4), and our survey covers the whole cluster area. As a consequence, in the following we will adopt $4^{\prime}$ as the cluster radius and refer to this 


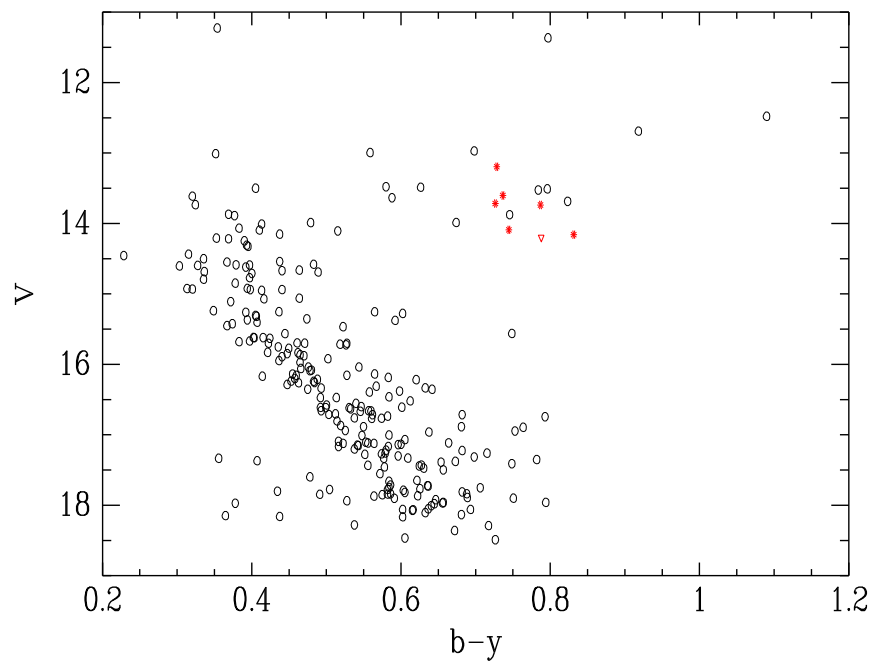

Figure 6. CMD of the cluster within $3: 5$ of the cluster center. Symbols have the same meaning as in Figure 3.

area as the cluster area, while the area outside $4^{\prime}$ from the cluster center will be referred to as the offset field.

\subsection{Photometric Reddening and Metallicity}

In the absence of membership information for any stars beyond those in Table 4, we can enhance the likelihood of including cluster members in our sample by restricting the analysis to stars within 3.5 of the cluster center, just short of the transition region from the cluster to the field based upon star counts. Figure 6 shows the $V, b-y$ CMD for stars within this core; all red giant members, independent of radial location, are plotted as stars, and open triangles are probable nonmembers. The cluster's turnoff region and the blue edge of the main sequence are well defined to the limit of the survey. The color spread at the top of the turnoff and the color and magnitude differential between the turnoff and the giant branch are very reminiscent of NGC 5822, a cluster of slightly subsolar metallicity with an age of $0.9 \mathrm{Gyr}$ (Carraro et al. 2011). An additional means of demonstrating the cluster population comes from the $V, h k \mathrm{CMD}$ for the core region, shown in Figure 7 . In the likely probability that there is a modest reddening range across the face of the cluster, this CMD minimizes the impact due to the weak sensitivity of $h k$ to reddening but a strong sensitivity to temperature and metallicity changes, factors we will make effective use of below. The tight vertical band defining the cluster turnoff reflects this fact, while the steep slope in $V$ with $h k$ is indicative of the cluster age; the trend in $h k$ with decreasing $b-y$ plateaus as the stellar sample moves from $\mathrm{F}$ to A stars, leading to an almost vertical turnoff in the $V, h k$ CMD for clusters of intermediate age, as illustrated by NGC 5822 in Figure 13 of Carraro et al. (2011). Among the giants, the positions of the two faintest radial-velocity members, 663 and 1349, place them redward of the majority of the probable clump stars and indicate that, despite their similar velocities, they are probable field stars. In the absence of more information, they will be retained in the discussions below.

To further isolate probable members for defining the reddening and metallicity, we restrict our sample to stars populating the blue edge of the cluster main sequence between $V=15.50$ and 17.0. The bright cutoff eliminates the evolved

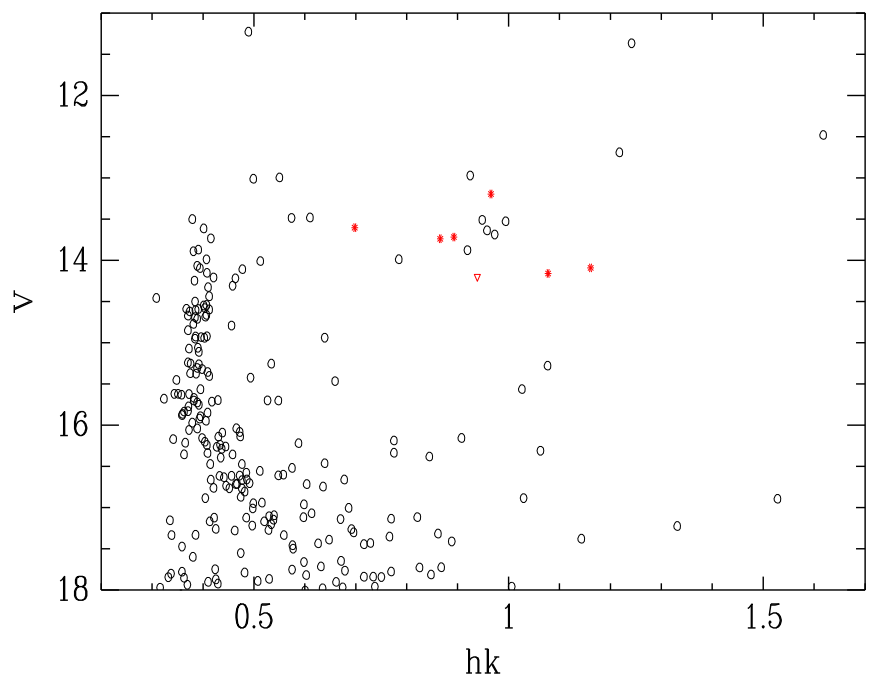

Figure 7. $V, h k \mathrm{CMD}$ of the cluster within 3.5 of the cluster center. Symbols have the same meaning as in Figure 6.

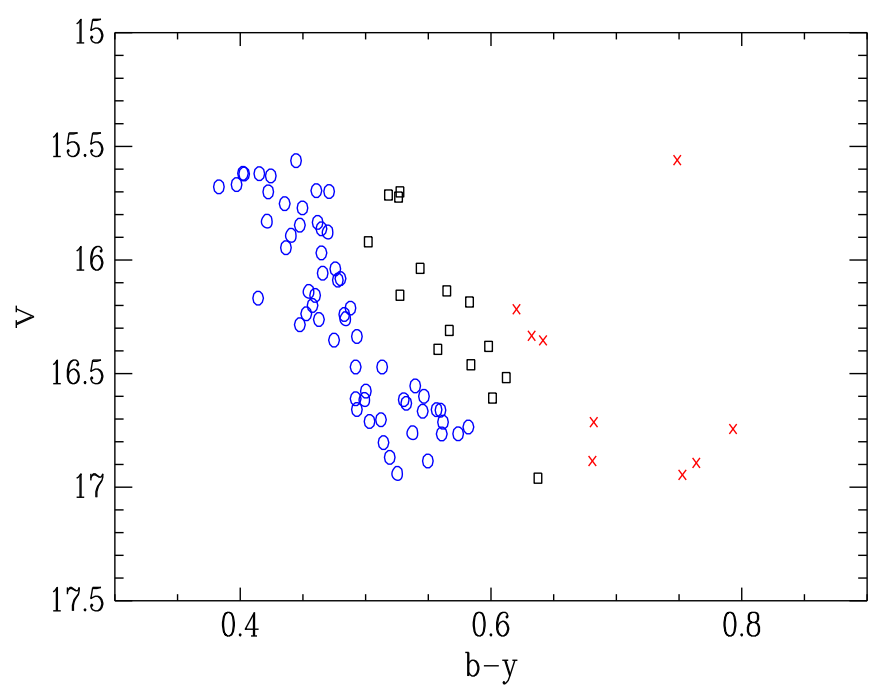

Figure 8. CMD of the cluster unevolved main sequence within 3!.5 of the cluster center. Blue open circles are probable single-star members, black squares are potential binaries, and red crosses are likely nonmembers.

stars that populate the color spread at the top of the main sequence, and the faint boundary defines the magnitude range where errors in the color indices begin to increase for $m_{1}, h k$, and especially $c_{1}$. The blue edge of the main sequence in this magnitude range was used to define a single-star boundary, and any star within $\sim 0.4$ mag of the boundary was classified as a single star (blue circles in Figure 8). Stars between 0.4 and $0.8 \mathrm{mag}$ were classed as probable binaries (black squares in Figure 8), if members, while all stars more than $0.8 \mathrm{mag}$ beyond the main sequence were tagged as likely field stars (red crosses in Figure 8).

We can check this classification using the $V, h k$ CMD of Figure 9. Stars can be located off the main sequence for a variety of reasons: bad or contaminated photometry, binarity, excessive reddening compared to the typical cluster star, or nonmembership. As noted earlier, the $h k$ index is very sensitive to color changes due to temperature and only weakly impacted by reddening. In fact, increased reddening moves a star blueward in $h k$. As shown in Figure 9, the separation by class as defined by Figure 8 is well corroborated. With only four 


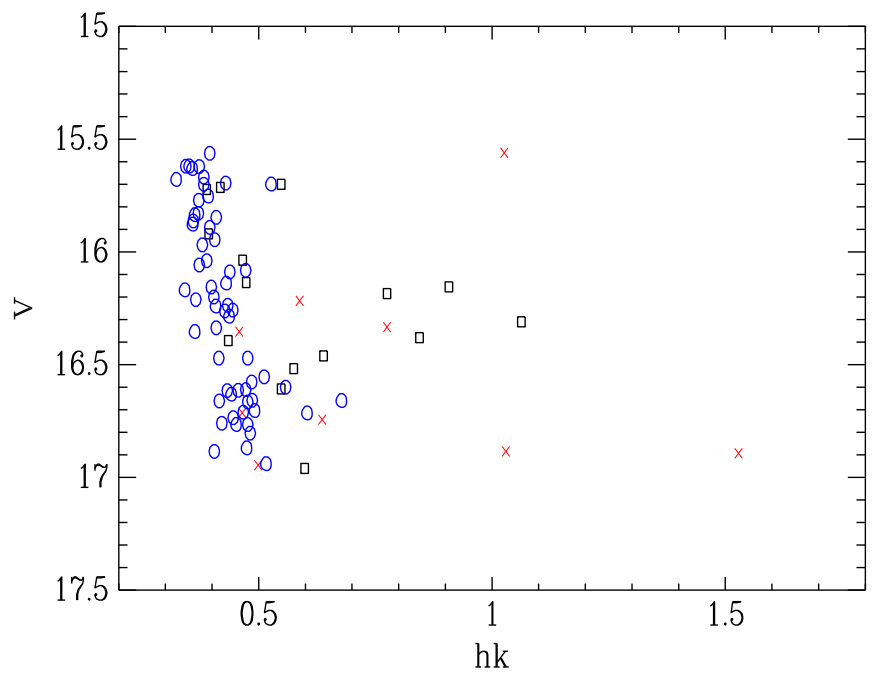

Figure 9. Same as Figure 8 using $h k$ as the temperature index.

obvious exceptions, the single stars form a well-defined turnoff band covering a modest range in $h k$. The stars redward of the main-sequence band in Figure 9 are dominated by the stars tagged as redder in Figure 8, indicating that these are truly redder than the cluster sequence and that the majority are probable nonmembers. Three red crosses that sit on the main sequence in Figure 9 deserve some explanation. These are either highly reddened field stars or, more likely, field stars in the direction of the cluster with significantly lower metallicity than Tombaugh 1. For the metallicity and reddening estimation, we will limit the sample to the single stars (blue circles), with the four points that deviate from the cluster main sequence in Figure 9 excluded.

For consistency with past cluster work, we will derive the reddening from two Strömgren relations from Olsen (1988) and Nissen (1988), slightly modified versions of the original relations derived by Crawford (1975, 1979). Reddening estimates are derived in an iterative fashion. The indices are corrected using an initial guess at the cluster reddening, and the intrinsic $b-y$ is derived using the reddening-free $\mathrm{H} \beta$ adjusted for metallicity and evolutionary state. A new reddening is derived by comparing the observed and intrinsic colors, and the procedure is repeated. The reddening estimate invariably converges after two to three iterations. To derive the reddening, one needs to correct $b-y$ for metallicity, so a fixed $[\mathrm{Fe} / \mathrm{H}]$ is adopted for the cluster and the reddening derived under a range of $[\mathrm{Fe} / \mathrm{H}]$ assumptions that bracket the final value. The complementary procedure is to vary the mean reddening value for the cluster and derive the mean $[\mathrm{Fe} / \mathrm{H}]$. Ultimately, only one combination of $E(b-y)$ and $[\mathrm{Fe} / \mathrm{H}]$ will be consistent.

For Tombaugh 1, the metallicity from $m_{1}$ was varied between $[\mathrm{Fe} / \mathrm{H}]=-0.28$ and +0.12 , generating a range of $E(b-y)=0.224$ to 0.214 for the relation of Olsen (1988) and 0.223-0.216 for Nissen (1988) from 51 stars within the $\mathrm{H} \beta$ calibration range. In all cases, the standard error of the mean for the final $E(b-y)$ is $\pm 0.006 \mathrm{mag}$. For $[\mathrm{Fe} / \mathrm{H}]$ from $m_{1}$ equal to -0.16 , the reddening from the two relations is virtually identical at $E(b-y)=0.221 \pm 0.006$; the difference between the two reddening values is statistically insignificant compared to the standard errors of the mean. If $E(b-y)=0.73^{*} E(B-V)$, the reddening estimate from the Strömgren data alone is $E(B-V)=0.303 \pm 0.008$. There

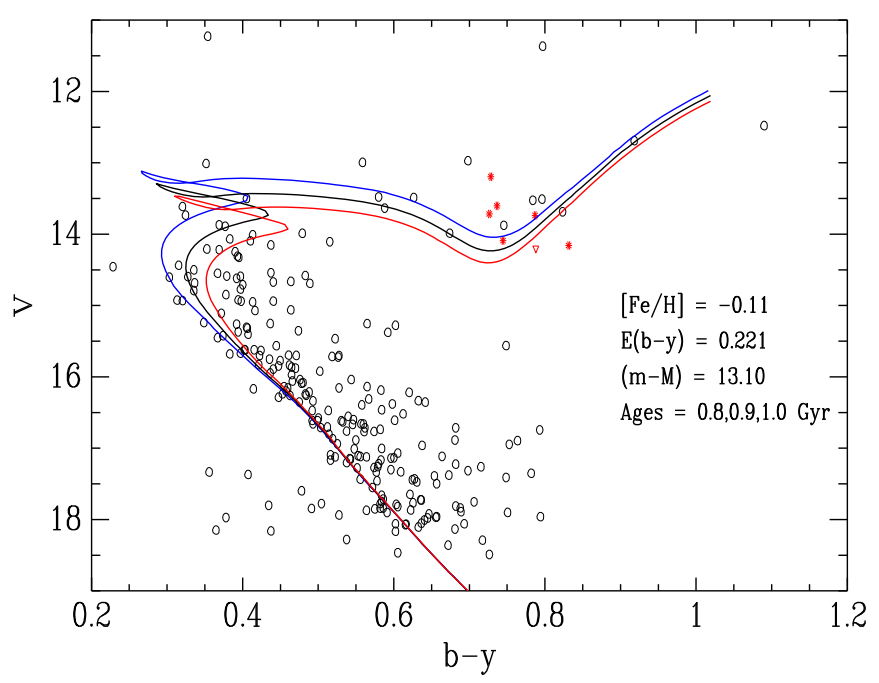

Figure 10. CMD of Figure 6 superposed on the VR isochrones with $[\mathrm{Fe} /$ $\mathrm{H}]=-0.11$, assuming $E(B-V)=0.303$ and $(m-M)=13.10$. The isochrones have ages of $0.8 \mathrm{Gyr}$ (blue), $0.9 \mathrm{Gyr}$ (black), and $1.0 \mathrm{Gyr}$ (red).

is weak evidence for a variation in $E(b-y)$ across the face of the cluster, with the reddening being higher on average by $0.03 \mathrm{mag}$ in the southwest and lower by a comparable amount in the northeast. Without more membership information, for the purposes of deriving the cluster parameters, we will adopt the cluster mean for all stars.

With the reddening set, we can derive the metallicity from both $m_{1}$ and $h k$, using $\mathrm{H} \beta$ as the primary temperature index. From 51 stars, $[\mathrm{Fe} / \mathrm{H}]=-0.165 \pm 0.027$ from $m_{1}$ and $-0.086 \pm 0.013$ from $h k$. If one anomalous measurement located more than three sigma from the cluster mean is removed from the $m_{1}$ analysis, the revised $[\mathrm{Fe} / \mathrm{H}]$ becomes $-0.153 \pm 0.025$. The greater uncertainty in the metallicity estimate from $m_{1}$ relative to $h k$ is a reflection of the greater sensitivity of $m_{1}$ to reddening changes and a lower sensitivity to metallicity variation; the small difference in $[\mathrm{Fe} / \mathrm{H}]$ can be entirely explained by a zero-point offset of 0.005 mag in $m_{1}$. Weighting the two photometric estimates by the inverse square of the errors leads to a final value of $[\mathrm{Fe} / \mathrm{H}]=-0.10 \pm 0.02$.

\subsection{Age and Distance Estimation}

One of the rare sets of available isochrones that include models transformed to the Strömgren system is the VictoriaRegina (VR) set of isochrones (VandenBerg et al. 2006). Figure 10 shows the scaled-solar models for $[\mathrm{Fe} / \mathrm{H}]=-0.11$, ages $0.8,0.9$, and $1.0 \mathrm{Gyr}$, adjusted for $E(b-y)=0.221$ and $(m-M)=13.10$. The symbols have the same meaning as in Figure 6. The already noted similarity of Tombaugh 1 to NGC 5822 is confirmed. In addition to the similar scatter in $b-y$ at the top of the turnoff, the best-fit age estimate is between 0.9 and $1.0 \mathrm{Gyr}$; the best fit to a different set of broadband isochones for NGC 5822 produced an age of $0.9 \pm 0.1 \mathrm{Gyr}$ for the more populated cluster (Carraro et al. 2011). The reddening-corrected true distance modulus is $(m-M)_{o}=12.15$, in excellent agreement with the most recent work of Kharchenko et al. (2013). 


\section{ATMOSPHERIC PARAMETERS AND ABUNDANCE ANALYSIS}

The equivalent-width measurements of absorption lines of $\mathrm{Na}, \mathrm{Mg}, \mathrm{Al}, \mathrm{Si}, \mathrm{Ca}, \mathrm{Ti}, \mathrm{Cr}, \mathrm{Ni}$, and $\mathrm{Fe}$ were used to obtain their respective chemical abundances, while the abundances of $\mathrm{Y}$, $\mathrm{Ba}, \mathrm{Ce}$, and $\mathrm{Nd}$ were derived through spectral synthesis. The equivalent widths were measured manually using the task SPLOT in IRAF to fit a Gaussian profile to the observed absorption line. We rejected the absorption lines with equivalent widths greater than $160 \mathrm{~m} \AA$ because these lines are saturated, which prevents a Gaussian fit to the absorption lines (Pereira et al. 2011). All equivalent widths used to obtain the atmospheric parameters and chemical abundances are shown in Tables 5-8.

The atomic-line list adopted in this work is the same as the one used by Santrich et al. (2013) and Sales Silva et al. (2014). For the Ba II line, the hyperfine structure (HFS) was taken into account, and we used the line list of Carraro et al. (2014c). In Tables 5 and 6 we describe the line list with excitation potential $(\xi)$ and oscillator strength $(g f)$ for the absorption lines of $\mathrm{Fe}_{\mathrm{I}}$ and $\mathrm{Fe}$ II. The values of the oscillator strength adopted for the $\mathrm{Fe}$ I and Fe II lines were taken from Lambert et al. (1996) and Castro et al. (1997). Tables 7 and 8 show the atomic parameters ( $g f$ and $\xi$ values) of the absorption lines of the elements $\mathrm{Na}$, $\mathrm{Mg}, \mathrm{Al}, \mathrm{Si}, \mathrm{Ca}, \mathrm{Ti}, \mathrm{Cr}$, and $\mathrm{Ni}$ with their respective references (column 5). The atomic parameters for several transitions of Ti, $\mathrm{Cr}$, and $\mathrm{Ni}$ were retrieved from the National Institute of Science and Technology Atomic Spectra Database (Martin et al. 2002). For Na we used only two absorption lines, 6154.226 and $6160.747 \AA$. These Na lines have a clean profile, which makes it possible to calculate the chemical abundance of Na through the equivalent width (Smiljanic 2012). The absorption lines used to obtain $s$-process elemental abundances were 5289 and $5402 \AA$ for $\mathrm{Y}, 5853 \AA$ for $\mathrm{Ba}, 5117$ and $5187 \AA$ for $\mathrm{Ce}$, and $4914 \AA$ for Nd.

The atmospheric parameters and chemical abundances were obtained in the same manner as in Pereira et al. (2011), Santrich et al. (2013), and Sales Silva et al. (2014) using the local thermodynamic equilibrium (LTE) model atmospheres of Kurucz (1993) and the spectral analysis code MOOG (Sneden 1973). The excitation equilibrium was used to derive the effective temperature $\left(T_{\text {eff }}\right)$, as defined by a zero slope of the trend between the iron abundance derived from the $\mathrm{Fe}_{\mathrm{I}}$ lines and the excitation potential of the measured lines. The microturbulent velocity was adjusted until both the strong and weak $\mathrm{Fe}_{\mathrm{I}}$ lines (represented by reduced equivalent width, $W_{\lambda} / \lambda$ ) gave the same abundance. Finally, the surface gravity was determined using the ionization equilibrium found from the equality of the abundances of $\mathrm{Fe}_{\mathrm{I}}$ and $\mathrm{Fe}$ II. The final adopted atmospheric parameters are given in Table 9.

The uncertainty in the slopes of the $\mathrm{Fe}_{\mathrm{I}}$ abundance versus excitation potential and $\mathrm{Fe}_{\mathrm{I}}$ abundance versus reduced equivalent width were used to derive the uncertainties in our adopted effective temperatures $\left(T_{\text {eff }}\right)$ and microturbulent velocities $(\xi)$, respectively. The standard deviation in $\log g$ was set by changing this parameter around the adopted solution until the difference between $\mathrm{Fe}_{\mathrm{I}}$ and $\mathrm{Fe}$ II mean abundance differed by exactly one standard deviation of the $\left[\mathrm{Fe}_{\mathrm{I}} / \mathrm{H}\right]$ mean value. We estimated typical uncertainties in atmospheric parameters of the order of $\pm 180 \mathrm{~K}, \pm 0.3 \mathrm{dex}$, and $\pm 0.3 \mathrm{~km} \mathrm{~s}^{-1}$ for $T_{\mathrm{eff}}, \log g$, and $\xi$, respectively.
We also calculated the photometric effective temperature and photometric gravity to compare with our spectroscopic temperature and gravity. Photometric temperatures were calculated using the calibration of Alonso et al. (1999) and our values of $(b-y)$ with $E(b-y)=0.221$. The photometric gravity for each star was obtained from the equation

$$
\begin{aligned}
\log g_{\star}= & \log \frac{M_{\star}}{M_{\odot}}+0.4\left(V-A_{V}+\mathrm{BC}\right) \\
& +4 \log T_{\text {eff }}-2 \log r(\mathrm{pc})-10.62 .
\end{aligned}
$$

where $T_{\text {eff }}$ is the photometric effective temperature and $M$ is the mass. Based upon the VR isochrones and an age of $0.95 \mathrm{Gyr}$, the typical mass for a star in the color range of the likely member red giants is $2.15 M_{\odot}$. The photometric data of Table 4 were combined with an adopted distance of $r=2700 \mathrm{pc}$ and bolometric corrections (BC) defined by the relations of Alonso et al. (1999). For the Sun we adopted $M_{\text {bol } \odot}=4.74 \mathrm{mag}$ (Bessell et al. 1998), $T_{\text {eff } \odot}=5700 \mathrm{~K}$, and $\log g_{\odot}=4.3 \mathrm{dex}$.

It should be emphasized that, for the nonmember stars, adoption of the cluster parameters for distance, reddening, and metallicity will likely generate discordant results when compared to the spectroscopic parameters. For the six probable members, the temperature difference, in the sense (spectroscopic-photometric), is $52 \pm 196 \mathrm{~K}$, while the residuals in $\log g$ are $0.22 \pm 0.33$, consistent with the probable uncertainties in the estimates from the spectra, discussed above, and from the photometry. The modest offsets in temperature and gravity between the spectroscopic and photometric approaches are typical of such comparisons. Different methods are known to produce systematic offsets from each other, but there is no consensus on the source of these offsets (e.g., Allende Prieto et al. 1999; Frebel et al. 2013).

The determination of the atmospheric parameters (Table 9) and the knowledge of the atomic parameters of the absorption lines enable us to obtain the chemical abundance by measuring the equivalent widths or by spectral synthesis. In the case of equivalent widths, MOOG uses atmospheric and atomic parameters, as well as equivalent width measurements, to calculate the chemical abundance. For spectral synthesis, as input for MOOG we supply the atmospheric and atomic parameters and an estimate of the chemical abundance of the elements that influence the absorption line studied. Thereafter MOOG generates a synthetic spectrum that is compared with the observed spectrum, iterating until we find a chemical abundance that makes the synthetic spectra and observed identical.

Tables 11 and 12 show the chemical abundances of $\mathrm{Na}, \mathrm{Al}$, Fe-peak, alpha, and $s$-process elements in the notation $[\mathrm{X} / \mathrm{Fe}]$ and its standard deviation. We analyzed a high-resolution FEROS solar spectrum to obtain the atmospheric parameters and solar abundance with the same methodology applied to red clump stars of Tombaugh 1 . We found the following values for solar atmospheric parameters: $\quad T_{\text {eff } \odot}=5700 \mathrm{~K}$, $\log g_{\odot}=4.3 \mathrm{dex}$, and $\xi_{\odot}=0.9 \mathrm{~km} \mathrm{~s}^{-1}$. Pavlenko et al. (2012) found similar values of $5777 \mathrm{~K}, 4.44 \mathrm{dex}$, and $0.8 \mathrm{~km} \mathrm{~s}^{-1}$ for the effective temperature, surface gravity, and microturbulent velocity, respectively. In Table 10 we show our solar chemical abundances together with those given by Grevesse \& Sauval (1998) and Asplund et al. (2009) for comparison. The adopted abundances for the elements analyzed in this work were normalized to our solar abundances. 
Table 5

Observed Fe I and Fe II Lines

\begin{tabular}{|c|c|c|c|c|c|c|c|c|}
\hline \multirow[b]{3}{*}{ Element } & \multirow[b]{3}{*}{$\lambda(\AA)$} & \multirow[b]{3}{*}{$\chi(\mathrm{eV})$} & \multirow[b]{3}{*}{$\log g f$} & \multicolumn{5}{|c|}{ Equivalent Widths (mÅ) } \\
\hline & & & & \multicolumn{5}{|c|}{ Star } \\
\hline & & & & 769 & 806 & 1110 & 1118 & 395 \\
\hline \multirow[t]{37}{*}{$\mathrm{Fe} I$} & 5159.06 & 4.28 & -0.650 & $\ldots$ & $\ldots$ & $\cdots$ & 83 & 110 \\
\hline & 5162.27 & 4.18 & 0.079 & 158 & 148 & $\cdots$ & $\cdots$ & 153 \\
\hline & 5198.71 & 2.22 & -2.140 & 132 & 140 & 107 & $\cdots$ & 125 \\
\hline & 5242.49 & 3.63 & -0.970 & 105 & $\cdots$ & $\cdots$ & $\cdots$ & 111 \\
\hline & 5250.21 & 0.12 & -4.920 & $\ldots$ & $\ldots$ & $\ldots$ & $\ldots$ & 136 \\
\hline & 5288.52 & 3.69 & -1.510 & $\ldots$ & 37 & 63 & 79 & 78 \\
\hline & 5307.36 & 1.61 & -2.970 & $\cdots$ & 94 & 111 & 125 & 133 \\
\hline & 5315.05 & 4.37 & -1.400 & $\cdots$ & $\cdots$ & 59 & $\cdots$ & 51 \\
\hline & 5321.11 & 4.43 & -1.190 & 60 & $\cdots$ & $\cdots$ & $\cdots$ & $\cdots$ \\
\hline & 5322.04 & 2.28 & -2.840 & $\cdots$ & 31 & 78 & $\ldots$ & 103 \\
\hline & 5364.87 & 4.45 & 0.230 & 136 & $\ldots$ & 126 & 150 & 143 \\
\hline & 5367.47 & 4.42 & 0.439 & 141 & $\cdots$ & 131 & 135 & 142 \\
\hline & 5373.71 & 4.47 & -0.710 & 85 & $\ldots$ & 70 & 77 & $\ldots$ \\
\hline & 5410.91 & 4.47 & 0.400 & $\cdots$ & $\cdots$ & 140 & $\cdots$ & $\cdots$ \\
\hline & 5417.03 & 4.42 & -1.530 & 53 & $\cdots$ & 33 & 39 & 61 \\
\hline & 5441.34 & 4.31 & -1.580 & 47 & 18 & 46 & 58 & 65 \\
\hline & 5522.45 & 4.21 & -1.400 & 67 & $\ldots$ & 47 & 58 & 60 \\
\hline & 5531.98 & 4.91 & -1.460 & 37 & $\cdots$ & 31 & 29 & $\cdots$ \\
\hline & 5554.90 & 4.55 & -0.380 & $\cdots$ & 73 & $\cdots$ & $\cdots$ & $\cdots$ \\
\hline & 5560.21 & 4.43 & -1.040 & 70 & $\ldots$ & 63 & 64 & 72 \\
\hline & 5567.39 & 2.61 & -2.560 & $\cdots$ & $\cdots$ & 76 & $\cdots$ & 110 \\
\hline & 5576.09 & 3.43 & -0.850 & 135 & $\cdots$ & $\cdots$ & $\cdots$ & $\cdots$ \\
\hline & 5633.95 & 4.99 & -0.120 & 96 & 68 & $\ldots$ & 81 & $\ldots$ \\
\hline & 5635.82 & 4.26 & -1.740 & 58 & $\cdots$ & $\cdots$ & 47 & 56 \\
\hline & 5638.26 & 4.22 & -0.720 & $\cdots$ & $\cdots$ & $\cdots$ & $\cdots$ & 96 \\
\hline & 5691.50 & 4.30 & -1.370 & $\cdots$ & 23 & 66 & 54 & 79 \\
\hline & 5705.47 & 4.30 & -1.360 & 64 & $\cdots$ & 68 & 57 & $\cdots$ \\
\hline & 5731.76 & 4.26 & -1.150 & $\cdots$ & $\cdots$ & $\cdots$ & $\cdots$ & 76 \\
\hline & 5806.73 & 4.61 & -0.900 & 73 & $\cdots$ & $\cdots$ & 65 & 62 \\
\hline & 5852.22 & 4.55 & -1.180 & 62 & $\cdots$ & 48 & $\cdots$ & 60 \\
\hline & 5883.82 & 3.96 & -1.210 & 90 & $\cdots$ & 69 & $\cdots$ & $\cdots$ \\
\hline & 5934.65 & 3.93 & -1.020 & 97 & $\cdots$ & $\cdots$ & $\cdots$ & 92 \\
\hline & 6020.17 & 4.61 & -0.210 & $\cdots$ & 101 & $\cdots$ & $\cdots$ & $\cdots$ \\
\hline & 6024.06 & 4.55 & -0.060 & $\ldots$ & $\ldots$ & 109 & 119 & 126 \\
\hline & 6027.05 & 4.08 & -1.090 & 103 & 44 & 80 & 87 & 95 \\
\hline & 6056.01 & 4.73 & -0.400 & 93 & $\cdots$ & 78 & 81 & 86 \\
\hline & 6065.48 & 2.61 & -1.530 & 157 & $\cdots$ & 125 & 154 & 156 \\
\hline \multirow[t]{25}{*}{$\mathrm{Fe}_{\mathrm{I}}$} & 6079.01 & 4.65 & -0.970 & $\cdots$ & $\cdots$ & $\cdots$ & 71 & $\cdots$ \\
\hline & 6096.66 & 3.98 & -1.780 & 56 & $\cdots$ & 50 & 49 & 62 \\
\hline & 6120.25 & 0.91 & -5.950 & $\cdots$ & $\cdots$ & 24 & $\cdots$ & $\cdots$ \\
\hline & 6151.62 & 2.18 & -3.290 & 92 & $\cdots$ & 75 & 90 & 92 \\
\hline & 6157.73 & 4.08 & -1.110 & 98 & 36 & 89 & 94 & 89 \\
\hline & 6165.36 & 4.14 & -1.470 & 59 & $\cdots$ & 59 & 73 & 59 \\
\hline & 6170.51 & 4.79 & -0.380 & $\cdots$ & 36 & $\cdots$ & $\cdots$ & $\cdots$ \\
\hline & 6173.34 & 2.22 & -2.880 & 107 & 39 & 92 & 112 & 122 \\
\hline & 6187.99 & 3.94 & -1.570 & 73 & $\cdots$ & 60 & 72 & 80 \\
\hline & 6200.31 & 2.60 & -2.440 & 118 & $\cdots$ & $\cdots$ & 107 & 103 \\
\hline & 6213.43 & 2.22 & -2.480 & 123 & $\cdots$ & $\cdots$ & $\cdots$ & $\cdots$ \\
\hline & 6265.13 & 2.18 & -2.550 & 128 & 95 & $\cdots$ & 132 & 135 \\
\hline & 6311.50 & 2.83 & -3.230 & $\cdots$ & $\cdots$ & $\cdots$ & 57 & $\ldots$ \\
\hline & 6322.69 & 2.59 & -2.430 & $\ldots$ & $\ldots$ & 88 & $\cdots$ & 106 \\
\hline & 6380.74 & 4.19 & -1.320 & 87 & $\cdots$ & 65 & 86 & 70 \\
\hline & 6392.54 & 2.28 & -4.030 & $\cdots$ & $\cdots$ & 29 & $\cdots$ & $\cdots$ \\
\hline & 6411.65 & 3.65 & -0.660 & 142 & 150 & 125 & 138 & $\cdots$ \\
\hline & 6421.35 & 2.28 & -2.010 & $\cdots$ & 152 & 135 & 153 & 150 \\
\hline & 6430.85 & 2.18 & -2.010 & 155 & $\cdots$ & 126 & 155 & 159 \\
\hline & 6436.41 & 4.19 & -2.460 & 25 & $\ldots$ & $\ldots$ & $\ldots$ & $\ldots$ \\
\hline & 6469.19 & 4.83 & -0.620 & 85 & 29 & 74 & 79 & 71 \\
\hline & 6593.87 & 2.44 & -2.420 & 124 & $\cdots$ & $\cdots$ & 117 & 129 \\
\hline & 6597.56 & 4.79 & -0.920 & 71 & $\ldots$ & $\ldots$ & 55 & 57 \\
\hline & 6608.03 & 2.28 & -4.030 & 48 & $\cdots$ & 28 & 39 & 37 \\
\hline & 6646.93 & 2.61 & -3.990 & 45 & $\ldots$ & $\ldots$ & $\ldots$ & 30 \\
\hline
\end{tabular}


Table 5

(Continued)

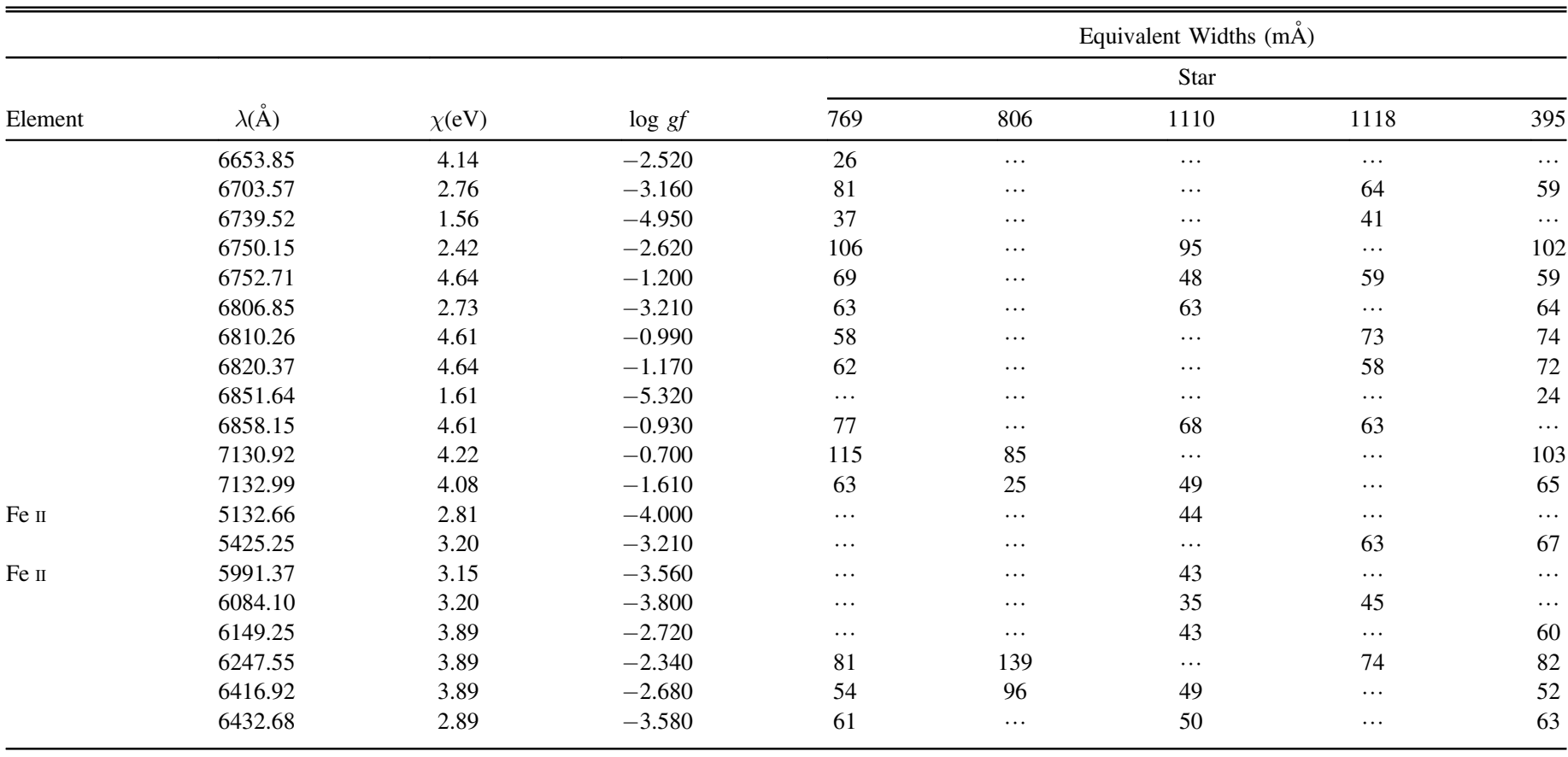

In the seventh row of Tables 11 and 12 we show the mean chemical abundances of Tombaugh 1 for each element with their respective standard deviations.

The approach to estimating the uncertainties in abundance consists of determining how the abundance for each element reacts to the errors associated with each atmospheric parameter, independent of the others. After that we combine quadratically all these errors and set this result as the total abundance uncertainty. These total uncertainties are given in the fifth column of Table 13 for star 769 . We chose the star 769 to determine the abundance uncertainties because it is one of the cluster giants that had the greatest number of elements with derived chemical abundance. The uncertainties for aluminum were not obtained for star 769 because their absorption lines are located in the spectral gaps, so we used star 663 to calculate the aluminum uncertainties. The uncertainties in abundance for the other stars generate similar values.

\section{RESULTS OF ABUNDANCE ANALYSIS}

In this section we discuss the results of our chemical analysis via comparison with the chemical abundances of field giant stars and open clusters from the literature.

\subsection{Metallicity and Iron-peak Elements}

In Table 9 we show the metallicities obtained for our giants. The range of metallicity for the six stars classified as members of Tombaugh 1 is -0.16 to $0.10 \mathrm{dex}$, with the mean of $-0.02 \pm 0.05$ dex. The spectroscopic values are consistent with a photometric value of $-0.10 \pm 0.02$. A weighted average of the two approaches gives a final $[\mathrm{Fe} / \mathrm{H}]=-0.08$ for Tombaugh 1. A comparison with past abundance estimates provides little insight given the large uncertainty in previous published estimates of this cluster parameter.

In Figures $11-13$ we show the abundance ratio of $[\mathrm{X} / \mathrm{Fe}]$ versus metallicity for our sample of giants, for giants from
Mishenina et al. (2006) and Luck \& Heiter (2007), and also for the open clusters NGC 6192, NGC 6404, and NGC 6583 (Magrini et al. 2010); NGC 3114 (Santrich et al. 2013); NGC 2527, NGC 2682, NGC 2482, NGC 2539, NGC 2335, NGC 2251, and NGC 2266 (Reddy et al. 2013); Trumpler 20 (Carraro et al. 2014c); NGC 4337 (Carraro et al. 2014b); NGC 4815 and NGC 6705 (Magrini et al. 2014); and Cr 110, Cr 261, NGC 2477, NGC 2506, and NGC 5822 (Mishenina et al. 2015). For the $s$-process elements of Figure 13, we added data of the open clusters Berkeley 25, Berkeley 73, Berkeley 75, Ruprecht 4, Ruprecht 7, NGC 6192, NGC 6404, and NGC 6583 from Mishenina et al. (2013).

From our chemical analysis of Tombaugh 1 we derive the following mean abundance ratios $[\mathrm{X} / \mathrm{Fe}]$ for $\mathrm{Cr}$ and $\mathrm{Ni}$ : $0.10 \pm 0.06$ and $-0.04 \pm 0.02$ dex, respectively. Our $[\mathrm{Cr} / \mathrm{Fe}]$ and $[\mathrm{Ni} / \mathrm{Fe}]$ of Tombaugh 1 are in good agreement with disk field giants and open clusters from the literature, as demonstrated in Figure 11.

\section{2. $\mathrm{Na}, \mathrm{Al}$, and Alpha Elements}

$\mathrm{Na}$ is synthesized during hydrostatic carbon-burning in massive stars and also through the $\mathrm{NeNa}$ cycle during $\mathrm{H}$-burning through the $\mathrm{CNO}$ cycle in intermediate-mass and massive stars (Denisenkov \& Denisenkova 1990; Woosley \& Weaver 1995). The chemical analysis of sodium must be performed taking into account non-local thermodynamic equilibrium (NLTE) effects, these effects being greater for higher equivalent widths and lower gravities (Gratton et al. 1999; Lind et al. 2011; Smiljanic 2012). In order to account for the NLTE effects in the $\mathrm{Na}$ abundances, we used the corrections of Gratton et al. (1999). These corrections were typically smaller than $0.10 \mathrm{dex}$, with higher values for giants with lower $\log g$ (stars 784 and 1534). With this NLTE correction, the range in the abundance ratio $[\mathrm{Na} / \mathrm{Fe}]$ for the red clump stars of Tombaugh 1 goes from 0.38 to 0.05 dex, with a mean value of $0.17 \pm 0.06$. Star 1534 , classified as a field 
Table 6

Observed $\mathrm{Fe} \mathrm{I}$ and $\mathrm{Fe}$ II Lines

\begin{tabular}{|c|c|c|c|c|c|c|c|c|}
\hline \multirow[b]{3}{*}{ Element } & \multirow[b]{3}{*}{$\lambda(\AA)$} & \multirow[b]{3}{*}{$\chi(\mathrm{eV})$} & \multirow[b]{3}{*}{$\log g f$} & \multicolumn{5}{|c|}{ Equivalent Widths (mÅ) } \\
\hline & & & & \multicolumn{5}{|c|}{ Star } \\
\hline & & & & 1534 & 1616 & 1349 & 784 & 663 \\
\hline \multirow[t]{37}{*}{$\mathrm{Fe}$ I } & 5159.06 & 4.28 & -0.650 & $\cdots$ & 98 & 106 & 109 & $\cdots$ \\
\hline & 5253.03 & 2.28 & -3.790 & $\cdots$ & $\ldots$ & $\ldots$ & $\ldots$ & 91 \\
\hline & 5288.52 & 3.69 & -1.510 & $\ldots$ & $\ldots$ & $\ldots$ & 93 & 116 \\
\hline & 5307.36 & 1.61 & -2.970 & $\cdots$ & 109 & 147 & 148 & $\cdots$ \\
\hline & 5315.05 & 4.37 & -1.400 & $\ldots$ & $\ldots$ & $\ldots$ & $\ldots$ & 70 \\
\hline & 5321.11 & 4.43 & -1.190 & $\ldots$ & $\ldots$ & $\ldots$ & $\ldots$ & 98 \\
\hline & 5322.04 & 2.28 & -2.840 & 121 & $\cdots$ & $\cdots$ & $\cdots$ & $\cdots$ \\
\hline & 5364.87 & 4.45 & 0.230 & 158 & $\ldots$ & 148 & 154 & $\ldots$ \\
\hline & 5367.47 & 4.42 & 0.439 & 157 & $\ldots$ & $\ldots$ & $\ldots$ & $\ldots$ \\
\hline & 5373.71 & 4.47 & -0.710 & 116 & $\ldots$ & 113 & 89 & 116 \\
\hline & 5417.03 & 4.42 & -1.530 & 53 & 52 & 60 & 61 & 80 \\
\hline & 5441.34 & 4.31 & -1.580 & 44 & 40 & 57 & $\cdots$ & $\cdots$ \\
\hline & 5522.45 & 4.21 & -1.400 & $\ldots$ & $\ldots$ & 68 & 71 & 92 \\
\hline & 5554.90 & 4.55 & -0.380 & $\ldots$ & 103 & 122 & $\ldots$ & $\ldots$ \\
\hline & 5560.21 & 4.43 & -1.040 & $\ldots$ & $\ldots$ & 104 & $\ldots$ & $\ldots$ \\
\hline & 5567.39 & 2.61 & -2.560 & 122 & $\ldots$ & $\cdots$ & $\ldots$ & 155 \\
\hline & 5576.09 & 3.43 & -0.850 & $\ldots$ & $\ldots$ & $\ldots$ & 154 & $\cdots$ \\
\hline & 5624.02 & 4.39 & -1.330 & $\ldots$ & $\ldots$ & $\ldots$ & 76 & 103 \\
\hline & 5633.95 & 4.99 & -0.120 & $\ldots$ & 85 & 103 & $\cdots$ & $\cdots$ \\
\hline & 5635.82 & 4.26 & -1.740 & $\ldots$ & $\ldots$ & $\ldots$ & $\ldots$ & 58 \\
\hline & 5638.26 & 4.22 & -0.720 & $\ldots$ & 84 & $\ldots$ & 106 & $\ldots$ \\
\hline & 5691.50 & 4.30 & -1.370 & 85 & 51 & $\ldots$ & 74 & 104 \\
\hline & 5705.47 & 4.30 & -1.360 & $\ldots$ & 46 & $\ldots$ & 59 & 93 \\
\hline & 5717.83 & 4.28 & -0.979 & 116 & $\ldots$ & 122 & $\ldots$ & $\ldots$ \\
\hline & 5806.73 & 4.61 & -0.900 & $\ldots$ & $\cdots$ & $\ldots$ & 65 & 99 \\
\hline & 5814.81 & 4.28 & -1.820 & $\ldots$ & $\ldots$ & $\ldots$ & $\ldots$ & 62 \\
\hline & 5852.22 & 4.55 & -1.180 & $\cdots$ & 63 & 78 & 70 & 95 \\
\hline & 5883.82 & 3.96 & -1.210 & 94 & $\ldots$ & $\ldots$ & 101 & $\cdots$ \\
\hline & 5916.25 & 2.45 & -2.990 & 104 & $\ldots$ & $\ldots$ & $\ldots$ & $\ldots$ \\
\hline & 5934.65 & 3.93 & -1.020 & 120 & $\ldots$ & 142 & 117 & 140 \\
\hline & 6024.06 & 4.55 & -0.060 & $\ldots$ & 102 & $\ldots$ & $\ldots$ & $\ldots$ \\
\hline & 6027.05 & 4.08 & -1.090 & $\ldots$ & 72 & $\ldots$ & $\ldots$ & $\ldots$ \\
\hline & 6056.01 & 4.73 & -0.400 & $\ldots$ & 91 & $\ldots$ & $\ldots$ & 123 \\
\hline & 6065.48 & 2.61 & -1.530 & $\cdots$ & 151 & $\ldots$ & $\cdots$ & $\ldots$ \\
\hline & 6096.66 & 3.98 & -1.780 & $\ldots$ & 62 & 95 & 71 & 83 \\
\hline & 6151.62 & 2.18 & -3.290 & $\cdots$ & 79 & $\cdots$ & $\ldots$ & $\cdots$ \\
\hline & 6157.73 & 4.08 & -1.110 & 120 & 95 & $\ldots$ & 102 & 120 \\
\hline \multirow[t]{25}{*}{$\mathrm{Fe}$ I } & 6165.36 & 4.14 & -1.470 & $\ldots$ & 56 & $\ldots$ & 75 & 97 \\
\hline & 6173.34 & 2.22 & -2.880 & $\ldots$ & 98 & $\ldots$ & $\cdots$ & 145 \\
\hline & 6187.99 & 3.94 & -1.570 & $\ldots$ & $\ldots$ & $\cdots$ & 81 & 102 \\
\hline & 6200.31 & 2.60 & -2.440 & $\ldots$ & 111 & 145 & 126 & $\ldots$ \\
\hline & 6265.13 & 2.18 & -2.550 & 155 & $\ldots$ & $\ldots$ & 143 & $\ldots$ \\
\hline & 6380.74 & 4.19 & -1.320 & $\ldots$ & 75 & $\ldots$ & $\ldots$ & 119 \\
\hline & 6392.54 & 2.28 & -4.030 & $\ldots$ & $\ldots$ & $\ldots$ & 65 & 84 \\
\hline & 6411.65 & 3.65 & -0.660 & $\cdots$ & 145 & $\ldots$ & 157 & $\ldots$ \\
\hline & 6421.35 & 2.28 & -2.010 & $\cdots$ & 133 & $\ldots$ & $\ldots$ & $\ldots$ \\
\hline & 6430.85 & 2.18 & -2.010 & $\ldots$ & 148 & $\ldots$ & $\ldots$ & $\ldots$ \\
\hline & 6436.41 & 4.19 & -2.460 & $\cdots$ & $\ldots$ & $\ldots$ & 21 & 42 \\
\hline & 6469.19 & 4.83 & -0.620 & 70 & 82 & 102 & 99 & 117 \\
\hline & 6551.68 & 0.99 & -5.790 & $\ldots$ & $\ldots$ & 56 & 41 & 65 \\
\hline & 6591.31 & 4.59 & -2.070 & $\ldots$ & $\cdots$ & $\cdots$ & 23 & $\cdots$ \\
\hline & 6593.87 & 2.44 & -2.420 & 137 & $\ldots$ & $\ldots$ & $\ldots$ & $\ldots$ \\
\hline & 6597.56 & 4.79 & -0.920 & 52 & 40 & $\cdots$ & 62 & 73 \\
\hline & 6608.03 & 2.28 & -4.030 & $\ldots$ & 40 & $\ldots$ & 66 & 76 \\
\hline & 6609.11 & 2.56 & -2.690 & $\ldots$ & $\ldots$ & 140 & $\ldots$ & $\ldots$ \\
\hline & 6646.93 & 2.61 & -3.990 & 41 & $\ldots$ & $\ldots$ & $\ldots$ & 63 \\
\hline & 6703.57 & 2.76 & -3.160 & $\ldots$ & $\ldots$ & 66 & 79 & $\ldots$ \\
\hline & 6739.52 & 1.56 & -4.950 & $\ldots$ & $\ldots$ & $\ldots$ & 70 & 48 \\
\hline & 6750.15 & 2.42 & -2.620 & $\cdots$ & $\cdots$ & 150 & $\cdots$ & $\ldots$ \\
\hline & 6752.71 & 4.64 & -1.200 & $\cdots$ & 38 & 86 & 73 & 101 \\
\hline & 6806.85 & 2.73 & -3.210 & $\ldots$ & 59 & 90 & 87 & 104 \\
\hline & 6810.26 & 4.61 & -0.990 & $\ldots$ & $\ldots$ & 98 & 91 & 94 \\
\hline
\end{tabular}


Table 6

(Continued)

\begin{tabular}{|c|c|c|c|c|c|c|c|c|}
\hline \multirow[b]{2}{*}{ Element } & \multirow[b]{2}{*}{$\lambda(\AA)$} & \multirow[b]{2}{*}{$\chi(\mathrm{eV})$} & \multirow[b]{2}{*}{$\log g f$} & \multicolumn{5}{|c|}{ Equivalent Widths (mÅ) } \\
\hline & & & & 1534 & 1616 & 1349 & 784 & 663 \\
\hline & 6858.15 & 4.61 & -0.930 & $\cdots$ & 65 & $\ldots$ & $\ldots$ & 103 \\
\hline & 7130.92 & 4.22 & -0.700 & $\cdots$ & $\ldots$ & $\ldots$ & 130 & $\ldots$ \\
\hline & 7132.99 & 4.08 & -1.610 & $\ldots$ & $\ldots$ & 77 & 64 & 90 \\
\hline & 6084.10 & 3.20 & -3.800 & $\ldots$ & 25 & $\ldots$ & $\ldots$ & $\cdots$ \\
\hline & 6149.25 & 3.89 & -2.720 & 73 & 54 & 80 & 63 & $\ldots$ \\
\hline & 6247.55 & 3.89 & -2.340 & $\ldots$ & 68 & $\ldots$ & 82 & 73 \\
\hline & 6416.92 & 3.89 & -2.680 & 78 & 63 & 69 & 59 & 56 \\
\hline & 6432.68 & 2.89 & -3.580 & $\ldots$ & $\ldots$ & $\ldots$ & 68 & 53 \\
\hline
\end{tabular}

giant, showed the strongest NLTE effects with a correction of 0.22 dex, mainly due to its low surface gravity $(\log g=2.0$ ).

Chemical mixtures in the stellar interior can significantly modify the surface $[\mathrm{Na} / \mathrm{Fe}]$ (e.g., Charbonnel \& Lagarde 2010). Comparing Tombaugh 1 with the models of Charbonnel \& Lagarde (2010), the mean cluster overabundance of $[\mathrm{Na} /$ $\mathrm{Fe}]=0.17$ among the giants is in excellent agreement with the values expected for models with thermohaline and rotationinduced mixing: $[\mathrm{Na} / \mathrm{Fe}]=0.18$ for $M=2.0 \quad M_{\odot}$ and rotational velocities of $110 \mathrm{~km} \mathrm{~s}^{-1}$ on the zero-age main sequence (ZAMS). The range of $[\mathrm{Na} / \mathrm{Fe}]$ among the giants of Tombaugh 1 could be explained by a range of rotation velocities among the stars in ZAMS that produced the giants (Charbonnel \& Lagarde 2010).

The production of $\mathrm{Al}, \mathrm{Mg}, \mathrm{Si}, \mathrm{Ca}$, and $\mathrm{Ti}$ occurs mainly in massive stars, whereas the production of the iron-peak elements is dominated by SN Ia (e.g., Woosley \& Weaver 1995; Iwamoto et al. 1999). Thus, the chemical ratio of $\mathrm{Al}$ and alpha elements with $\mathrm{Fe}$ can give us important information about the SN Ia and SN II contributions to the galactic components (bulge, disk, and halo). The mean abundances of $\mathrm{Mg}, \mathrm{Si}$, and $\mathrm{Ca}$ relative to $\mathrm{Fe}$ for Tombaugh 1 show essentially solar values of $+0.03 \pm 0.05,+0.01 \pm 0.07$, and $+0.01 \pm 0.03$, respectively. In the case of $[\mathrm{Ti} / \mathrm{Fe}]$, we found for Tombaugh 1 a slightly overabundant value relative to the Sun with a mean of $+0.11 \pm 0.04$ dex. Our values of $[\mathrm{X} / \mathrm{Fe}]$ for alpha elements in Tombaugh 1 are consistent with the disk giants of Luck \& Heiter (2007) and Mishenina et al. (2006) and also with open clusters with similar metallicity from the literature (Figure 12). The decay of the $[\mathrm{X} / \mathrm{Fe}]$ ratio to alpha elements with increasing metallicity in the disk stars, as observed in Figure 12, can be explained by the SN Ia yields (Iwamoto et al. 1999), i.e., by high creation of $\mathrm{Fe}$ and low generation of alpha elements. [Al/ Fe] for Tombaugh 1 is similar to [Ti/Fe], with a mean of +0.15 , in agreement with the chemical pattern of $\mathrm{Al}$ in the galactic disk (Figure 12).

\subsection{Neutron-capture Elements}

The elements $\mathrm{Y}, \mathrm{Ba}, \mathrm{Ce}$, and $\mathrm{Nd}$ are formed mainly in the stellar interior by a slow neutron-capture process ( $s$-process) during the asymptotic giant branch (AGB) phase and are transported to the stellar surface by the third dredge-up (Busso et al. 1999). In Tombaugh 1, the light $s$-process element, Y, has a near-solar $[\mathrm{X} / \mathrm{Fe}]$ mean of $+0.06 \pm 0.04 \mathrm{dex}$, while $[\mathrm{X} / \mathrm{Fe}]$ for heavy $s$-process elements ( $\mathrm{Ba}, \mathrm{Ce}$, and $\mathrm{Nd}$ ) shows an excess compared to the Sun, with a mean of $+0.35 \pm 0.03$ for $\mathrm{Ba}$, $+0.25 \pm 0.06$ for $\mathrm{Ce}$, and $+0.37 \pm 0.05$ for $\mathrm{Nd}$. The difference between light and heavy $s$-process elements is an indicator of $s$-process efficiency (e.g., Luck \& Bond 1981, 1991; Busso et al. 2001; Pereira et al. 2011), implying a high $s$ process efficiency for Tombaugh 1. Other open clusters exhibiting this same behavior include the Hyades (De Silva et al. 2006); Berkeley 18, Berkeley 21, Berkeley 22, and Berkeley 32 (Yong et al. 2012); and Ruprecht 4, Ruprecht 7, NGC 6192, and NGC 6404 (Mishenina et al. 2013), among others. The $s$-process efficiency is an important observational constraint to stellar evolutionary models (e.g., Busso et al. 2001) and is affected by metallicity, stellar mass, and rotational velocity (e.g., Herwig et al. 2003; Lugaro et al. 2003).

Abundance measurements for $s$-process elements from the literature are highly inhomogeneous and difficult to compare with our results, which is due to the use of different absorption lines, atomic parameters, and analysis methods (see, e.g., Yong et al. 2012 for a detailed discussion). Nevertheless, our $s$ process abundances for Tombaugh 1 agree with the published $s$-process abundances for open clusters, as shown in Figure 13. Only our neodymium abundances show a slight overabundance with respect to open clusters and disk field giants from the literature.

\subsection{The Peculiar Tombaugh 1 Field Cepheid XZ CMa}

XZ CMa (star 806 in Table 4) is a short-period Cepheid $(P=2$.56; Caldwell \& Coulson 1987) situated within the coronal region of Tombaugh 1 but classified as not a cluster member (see Section 3.2). Three papers in the literature analyzed in detail the Cepheid XZ CMa (Turner 1983; Diethelm 1990; Yong et al. 2006). Turner (1983) and Diethelm (1990) conducted a photometric analysis of XZ CMa, while Yong et al. (2006) analyzed XZ CMa with high-resolution spectroscopy. Turner (1983), via $U B V$ photoelectric photometry, defined XZ CMa as an unlikely member of Tombaugh 1 and found that XZ CMa probably has an unresolved blue companion that is approximately $2.5 \mathrm{mag}$ fainter in $V$ because the phase of the minimum in the $U-V$ curve is shifted from the phase of minimum light by roughly $0.2-0.3$ of the star's period. Subsequently, based on the Walraven $V B L U W$ photometric system, Diethelm (1990) derived the mean atmospheric parameters $\left(T_{\mathrm{eff}}, \log g\right.$, and $\left.[\mathrm{Fe} / \mathrm{H}]\right)$ of $\mathrm{XZ} \mathrm{CMa,} \mathrm{obtaining}$ 
Table 7

Other Lines Studied

\begin{tabular}{|c|c|c|c|c|c|c|c|c|c|}
\hline \multirow[b]{3}{*}{ Element } & \multirow[b]{3}{*}{$\lambda$} & \multirow[b]{3}{*}{$\chi(\mathrm{eV})$} & \multirow[b]{3}{*}{$\log g f$} & \multirow[b]{3}{*}{ References } & \multicolumn{5}{|c|}{ 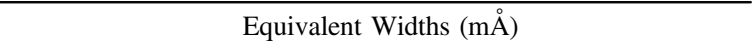 } \\
\hline & & & & & \multicolumn{5}{|c|}{ Star } \\
\hline & & & & & 769 & 806 & 1110 & 1118 & 395 \\
\hline $\mathrm{Na} I$ & 6154.22 & 2.10 & -1.51 & PS & 59 & 17 & 63 & 72 & 55 \\
\hline $\mathrm{Na} I$ & 6160.75 & 2.10 & -1.21 & R03 & 89 & $\ldots$ & 72 & 86 & 78 \\
\hline $\mathrm{Mg}_{\mathrm{I}}$ & 4730.04 & 4.34 & -2.39 & R03 & 81 & $\ldots$ & $\ldots$ & $\ldots$ & $\ldots$ \\
\hline $\mathrm{Mg}_{\mathrm{I}}$ & 5711.10 & 4.34 & -1.75 & R99 & 115 & 77 & 107 & $\ldots$ & $\cdots$ \\
\hline $\mathrm{Mg}_{\mathrm{I}}$ & 6318.71 & 5.11 & -1.94 & $\mathrm{Ca} 07$ & $\ldots$ & $\ldots$ & 45 & 58 & 55 \\
\hline $\mathrm{Mg}_{\mathrm{I}}$ & 6965.41 & 5.75 & -1.72 & MR94 & $\ldots$ & $\ldots$ & $\ldots$ & 43 & 40 \\
\hline $\mathrm{Mg}_{\mathrm{I}}$ & 7387.70 & 5.75 & -0.87 & MR94 & 85 & $\cdots$ & 105 & $\cdots$ & 80 \\
\hline $\mathrm{Mg}_{\mathrm{I}}$ & 8717.83 & 5.91 & -0.97 & WSM & 62 & $\ldots$ & $\ldots$ & 83 & $\ldots$ \\
\hline $\mathrm{Mg}_{\mathrm{I}}$ & 8736.04 & 5.94 & -0.34 & WSM & $\ldots$ & $\ldots$ & $\ldots$ & 128 & $\ldots$ \\
\hline $\mathrm{Si}$ I & 5793.08 & 4.93 & -2.06 & R03 & 52 & 17 & $\ldots$ & 61 & 63 \\
\hline Si I & 6125.03 & 5.61 & -1.54 & E93 & 44 & 24 & 29 & 39 & 42 \\
\hline Si I & 6131.58 & 5.62 & -1.68 & E93 & $\ldots$ & 14 & $\ldots$ & $\ldots$ & $\ldots$ \\
\hline Si I & 6155.14 & 5.62 & -0.77 & E93 & 91 & 77 & $\ldots$ & 87 & 89 \\
\hline $\mathrm{Si}$ I & 7800.00 & 6.18 & -0.72 & E93 & 69 & $\ldots$ & 58 & $\ldots$ & 68 \\
\hline $\mathrm{Si}_{\mathrm{I}}$ & 8728.01 & 6.18 & -0.36 & E93 & 89 & $\ldots$ & $\ldots$ & $\ldots$ & $\ldots$ \\
\hline $\mathrm{Ca}$ I & 6102.73 & 1.88 & -0.79 & D2002 & $\ldots$ & $\ldots$ & $\ldots$ & 149 & 141 \\
\hline $\mathrm{Ca}$ I & 6161.30 & 2.52 & -1.27 & E93 & 93 & $\ldots$ & 78 & 92 & 97 \\
\hline $\mathrm{Ca}$ I & 6166.44 & 2.52 & -1.14 & R03 & 83 & 43 & 88 & 99 & 86 \\
\hline $\mathrm{Ca}$ I & 6169.04 & 2.52 & -0.80 & $\mathrm{R} 03$ & 114 & $\ldots$ & 93 & 113 & 112 \\
\hline $\mathrm{Ca}$ I & 6169.56 & 2.53 & -0.48 & DS91 & 126 & $\ldots$ & 121 & 125 & 127 \\
\hline $\mathrm{Ca}$ I & 6455.60 & 2.51 & -1.29 & R03 & 79 & $\ldots$ & 71 & $\ldots$ & 89 \\
\hline $\mathrm{Ca} I$ & 6471.66 & 2.51 & -0.69 & S86 & 132 & 98 & $\ldots$ & $\ldots$ & 125 \\
\hline $\mathrm{Ca} I$ & 6493.79 & 2.52 & -0.11 & DS91 & 152 & $\ldots$ & $\ldots$ & $\ldots$ & $\ldots$ \\
\hline $\mathrm{Ti}_{\mathrm{I}}$ & 4534.78 & 0.84 & 0.280 & D2002 & 149 & $\ldots$ & $\ldots$ & $\ldots$ & $\ldots$ \\
\hline $\mathrm{Ti}_{\mathrm{I}}$ & 4758.12 & 2.25 & 0.420 & MFK & 80 & $\ldots$ & $\ldots$ & $\ldots$ & $\ldots$ \\
\hline Ti I & 4759.28 & 2.25 & 0.514 & MFK & 81 & $\ldots$ & $\ldots$ & $\ldots$ & $\ldots$ \\
\hline Ti I & 4820.41 & 1.50 & -0.439 & MFK & 83 & $\ldots$ & $\ldots$ & $\ldots$ & 83 \\
\hline Ti I & 4999.51 & 0.83 & 0.250 & MFK & $\ldots$ & $\ldots$ & 119 & $\ldots$ & $\ldots$ \\
\hline Ti I & 5009.66 & 0.02 & -2.259 & MFK & $\ldots$ & $\ldots$ & $\ldots$ & 71 & $\ldots$ \\
\hline $\mathrm{Ti}_{\mathrm{I}}$ & 5022.87 & 0.83 & -0.434 & MFK & $\ldots$ & $\ldots$ & $\ldots$ & $\ldots$ & 109 \\
\hline Ti I & 5039.96 & 0.02 & -1.130 & MFK & 118 & $\ldots$ & 96 & $\ldots$ & $\ldots$ \\
\hline $\mathrm{Ti}_{\mathrm{I}}$ & 5043.59 & 0.84 & -1.733 & MFK & 63 & $\ldots$ & $\ldots$ & $\ldots$ & $\ldots$ \\
\hline $\mathrm{Ti}_{\mathrm{I}}$ & 5087.06 & 1.43 & -0.840 & E93 & 60 & $\ldots$ & $\ldots$ & 56 & 56 \\
\hline Ti I & 5113.45 & 1.44 & -0.880 & E93 & $\ldots$ & $\ldots$ & $\ldots$ & 59 & $\ldots$ \\
\hline $\mathrm{Ti}_{\mathrm{I}}$ & 5145.47 & 1.46 & -0.574 & MFK & 82 & $\ldots$ & 62 & $\ldots$ & 67 \\
\hline Ti I & 5147.48 & 0.00 & -2.012 & MFK & 82 & $\ldots$ & $\ldots$ & 97 & 94 \\
\hline Ti I & 5173.75 & 0.00 & -1.120 & MFK & $\ldots$ & $\ldots$ & $\ldots$ & 136 & $\ldots$ \\
\hline Ti I & 5210.39 & 0.05 & -0.883 & MFK & $\ldots$ & $\ldots$ & 102 & $\ldots$ & $\ldots$ \\
\hline Ti I & 5219.71 & 0.02 & -2.292 & MFK & 81 & $\ldots$ & $\ldots$ & $\ldots$ & 79 \\
\hline Ti I & 5223.63 & 2.09 & -0.559 & MFK & $\ldots$ & $\ldots$ & 29 & $\ldots$ & $\ldots$ \\
\hline Ti I & 5295.78 & 1.05 & -1.633 & MFK & $\ldots$ & $\ldots$ & $\ldots$ & 39 & 44 \\
\hline $\mathrm{Ti}_{\mathrm{I}}$ & 5490.16 & 1.46 & -0.937 & MFK & 70 & $\ldots$ & $\ldots$ & $\ldots$ & $\ldots$ \\
\hline Ti I & 5689.48 & 2.30 & -0.469 & MFK & 34 & $\ldots$ & 35 & $\ldots$ & 40 \\
\hline $\mathrm{Ti}_{\mathrm{I}}$ & 5866.46 & 1.07 & -0.871 & E93 & $\ldots$ & $\ldots$ & 77 & 82 & 93 \\
\hline $\mathrm{Ti}_{\mathrm{I}}$ & 5922.12 & 1.05 & -1.465 & MFK & 62 & $\ldots$ & $\ldots$ & 59 & $\ldots$ \\
\hline Ti I & 5978.55 & 1.87 & -0.496 & MFK & 63 & $\ldots$ & 52 & 50 & $\ldots$ \\
\hline Ti I & 6091.18 & 2.27 & -0.370 & R03 & 43 & $\ldots$ & $\ldots$ & 42 & $\ldots$ \\
\hline $\mathrm{Ti}_{\mathrm{I}}$ & 6126.22 & 1.07 & -1.370 & R03 & 61 & 10 & 45 & $\ldots$ & 60 \\
\hline Ti I & 6258.11 & 1.44 & -0.355 & MFK & 99 & $\ldots$ & 81 & 81 & $\ldots$ \\
\hline Ti I & 6261.11 & 1.43 & -0.480 & B86 & 96 & $\ldots$ & 76 & 83 & 89 \\
\hline Ti I & 6554.24 & 1.44 & -1.219 & MFK & $\ldots$ & $\ldots$ & $\ldots$ & 55 & $\ldots$ \\
\hline $\mathrm{Cr}_{\mathrm{I}}$ & 4836.85 & 3.10 & -1.137 & MFK & 40 & $\ldots$ & $\ldots$ & $\ldots$ & 32 \\
\hline $\mathrm{Cr}_{\mathrm{I}}$ & 5200.18 & 3.38 & -0.650 & MFK & 40 & $\ldots$ & $\ldots$ & $\ldots$ & $\ldots$ \\
\hline $\mathrm{Cr}_{\mathrm{I}}$ & 5296.70 & 0.98 & -1.390 & GS & 133 & 82 & $\ldots$ & 134 & 132 \\
\hline $\mathrm{Cr}$ I & 5304.18 & 3.46 & -0.692 & MFK & 35 & $\ldots$ & $\ldots$ & $\ldots$ & $\ldots$ \\
\hline $\mathrm{Cr}$ I & 5345.81 & 1.00 & -0.980 & GS & $\ldots$ & 145 & 140 & $\ldots$ & $\ldots$ \\
\hline $\mathrm{Cr}$ I & 5348.32 & 1.00 & -1.290 & GS & $\ldots$ & 85 & 118 & 135 & 147 \\
\hline $\mathrm{Cr}$ I & 5783.07 & 3.32 & -0.500 & MFK & 49 & $\ldots$ & 47 & 60 & 62 \\
\hline $\mathrm{Cr}_{\mathrm{I}}$ & 5783.87 & 3.32 & -0.290 & GS & 74 & $\ldots$ & 64 & 68 & 76 \\
\hline $\mathrm{Cr} \mathrm{I}_{\mathrm{H}}$ & 5787.93 & 3.32 & -0.080 & GS & 71 & $\ldots$ & 69 & $\ldots$ & 70 \\
\hline $\mathrm{Cr}_{\mathrm{I}}$ & 6330.09 & 0.94 & -2.920 & R03 & $\ldots$ & $\ldots$ & 58 & 61 & 70 \\
\hline $\mathrm{Ni} I$ & 4904.42 & 3.54 & -0.170 & MFK & 116 & $\ldots$ & 102 & 100 & $\ldots$ \\
\hline
\end{tabular}


Table 7

(Continued)

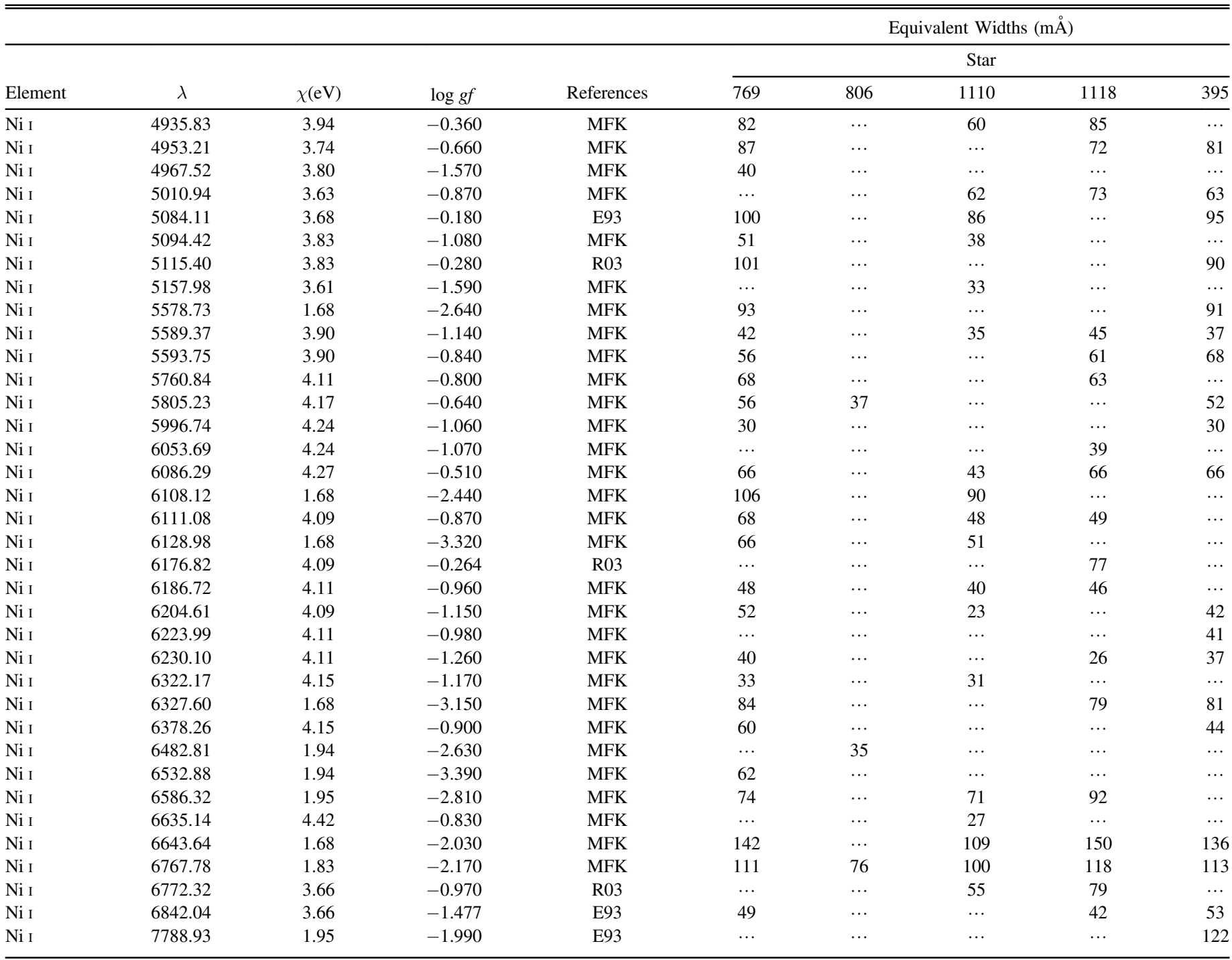

References. B86: Blackwell et al. (1986), Ca07: Carretta et al. (2007), D2002: Depagne et al. (2002), DS91: Drake \& Smith (1991), E93: Edvardsson et al. (1993), GS: Gratton \& Sneden (1988), MFK: Martin et al. (2002), MR94: McWilliam \& Rich (1994), PS: Preston \& Sneden (2001), R03: Reddy et al. (2003), R99: Reddy et al. (1999), WSM: Wiese et al. (1969).

$T_{\text {eff }}=6000 \mathrm{~K}, \quad \log \quad g=2.3 \quad(\mathrm{dex}), \quad$ and $\quad[\mathrm{Fe} /$ $\mathrm{H}]=-0.50 \pm 0.10$, with differences between our atmospheric parameters and Diethelm (1990) values of $\Delta T_{\mathrm{eff}}=0 \mathrm{~K}$, $\Delta \log g=0.4(\mathrm{dex})$, and $\Delta[\mathrm{Fe} / \mathrm{H}]=0.03(\mathrm{dex})$.

Lastly, Yong et al. (2006) determined the atmospheric parameters and the chemical abundances of three alpha elements ( $\mathrm{Si}, \mathrm{Ca}$, and $\mathrm{Ti}$ II) of $\mathrm{XZ} \mathrm{CMa,} \mathrm{using} \mathrm{the} \mathrm{same} \mathrm{method} \mathrm{but} \mathrm{a}$ different line list than used in this work. Our atmospheric parameters $T_{\text {eff }}, \log g$, and $\xi$ exhibit different values from those found by Yong et al. (2006), with differences of $750 \mathrm{~K}, 1.12$ (dex), and $1.58 \mathrm{~km} \mathrm{~s}^{-1}$, respectively. However, we and Yong et al. (2006) obtained similiar values of metallicity to XZ CMa $(\Delta[\mathrm{Fe} / \mathrm{H}]=0.04)$. Probably, the differences between $T_{\text {eff }}, \log g$, and $\xi$ displayed in this work and in Yong et al. (2006) are due to the observation of Cepheid XZ CMa in a distinct pulsation phase, which causes the determination of different values of atmospheric parameters $\left(T_{\mathrm{eff}}, \log g\right.$, and $\left.\xi\right)$ and similar metallicity. Finally, in both studies an overabundance of alpha elements in XZ CMa was found, with the mean of alpha elements in our analysis of $[\alpha /$ $\mathrm{Fe}]=0.13$ and in Yong et al. (2006) of $[\alpha / \mathrm{Fe}]=0.21$, characteristic of Cepheid stars in the outer disk (e.g., see Figure 15 of Yong et al. 2006).

Our results show that Cepheid XZ CMa has a chemical pattern similar to that presented by disk field stars and open clusters (see Figures 11 and 12). However, in Figure 13 we note that the Tombaugh 1 field star XZ CMa exhibits a high overabundance of Ba compared with field giants from the literature. To demonstrate the high $\mathrm{Ba}$ abundance in this star, in Figure 14 we present the observed and synthetic spectra in the region around the absorption line of Ba II $5853 \AA$ A. Classic Cepheids, like XZ $\mathrm{CMa}$, are not expected to present a high overabundance of $s$ process elements, like $\mathrm{Ba}$, because such stars are not evolved to the AGB; for example, Cepheids FO Cas, EW Aur, EE Mon, and FF Aur with metallicity similar to XZ CMa present the $[\mathrm{Ba} / \mathrm{Fe}]$ 
Table 8

Other Lines Studied

\begin{tabular}{|c|c|c|c|c|c|c|c|c|c|}
\hline \multirow[b]{3}{*}{ Element } & \multirow[b]{3}{*}{$\lambda$} & \multirow[b]{3}{*}{$\chi(\mathrm{eV})$} & \multirow[b]{3}{*}{$\log g f$} & \multirow[b]{3}{*}{ References } & \multicolumn{5}{|c|}{ Equivalent Widths $(\mathrm{m} \AA$ ) } \\
\hline & & & & & \multicolumn{5}{|c|}{ Star } \\
\hline & & & & & 1534 & 1616 & 1349 & 784 & 663 \\
\hline $\mathrm{Na} \mathrm{I}$ & 6154.22 & 2.10 & -1.51 & PS & 47 & 68 & $\ldots$ & 68 & 105 \\
\hline $\mathrm{Na}$ I & 6160.75 & 2.10 & -1.21 & R03 & $\cdots$ & $\cdots$ & $\cdots$ & 86 & 122 \\
\hline $\mathrm{Mg}_{\mathrm{I}}$ & 4730.04 & 4.34 & -2.39 & $\mathrm{R} 03$ & 88 & $\cdots$ & $\cdots$ & 79 & $\cdots$ \\
\hline $\mathrm{Mg}_{\mathrm{I}}$ & 5711.10 & 4.34 & -1.75 & R99 & 140 & 124 & 124 & 138 & 152 \\
\hline $\mathrm{Mg}_{\mathrm{I}}$ & 6318.71 & 5.11 & -1.94 & $\mathrm{Ca} 07$ & $\ldots$ & 58 & $\cdots$ & 74 & 91 \\
\hline $\mathrm{Mg}_{\mathrm{I}}$ & 7387.70 & 5.75 & -0.87 & MR94 & $\cdots$ & 102 & 98 & 113 & 120 \\
\hline $\mathrm{Mg}_{\mathrm{I}}$ & 8736.04 & 5.94 & -0.34 & WSM & 133 & $\ldots$ & $\cdots$ & $\ldots$ & $\ldots$ \\
\hline $\mathrm{Al}$ I & 6698.67 & 3.14 & -1.63 & R03 & $\ldots$ & $\ldots$ & 58 & $\ldots$ & $\ldots$ \\
\hline $\mathrm{Al}_{\mathrm{I}}$ & 7835.32 & 4.04 & -0.58 & R03 & $\ldots$ & 39 & $\ldots$ & 69 & 78 \\
\hline $\mathrm{Al}$ I & 7836.13 & 4.02 & -0.40 & R03 & $\ldots$ & 42 & $\ldots$ & 80 & 102 \\
\hline $\mathrm{Al}_{\mathrm{I}}$ & 8772.88 & 4.02 & -0.25 & R03 & 99 & $\ldots$ & 99 & $\ldots$ & $\ldots$ \\
\hline $\mathrm{Al}$ I & 8773.91 & 4.02 & -0.07 & R03 & 114 & $\ldots$ & $\ldots$ & $\ldots$ & 140 \\
\hline Si I & 5793.08 & 4.93 & -2.06 & R03 & 53 & 32 & 48 & 76 & 86 \\
\hline $\mathrm{Si}$ I & 6125.03 & 5.61 & -1.54 & E93 & 31 & 26 & $\ldots$ & 50 & $\ldots$ \\
\hline Si I & 6145.02 & 5.61 & -1.43 & E93 & $\ldots$ & $\ldots$ & $\ldots$ & 59 & 64 \\
\hline Si I & 6155.14 & 5.62 & -0.77 & E93 & 79 & 81 & $\ldots$ & 96 & $\ldots$ \\
\hline Si I & 7800.00 & 6.18 & -0.72 & E93 & 40 & $\cdots$ & $\ldots$ & 75 & $\ldots$ \\
\hline $\mathrm{Si}$ I & 8728.01 & 6.18 & -0.36 & E93 & 75 & $\ldots$ & $\ldots$ & $\ldots$ & 122 \\
\hline Si I & 8742.45 & 5.87 & -0.51 & E93 & $\ldots$ & $\ldots$ & 106 & $\ldots$ & 119 \\
\hline $\mathrm{Ca}$ I & 6102.73 & 1.88 & -0.79 & D2002 & $\ldots$ & $\ldots$ & $\ldots$ & 160 & $\ldots$ \\
\hline $\mathrm{Ca} I$ & 6161.30 & 2.52 & -1.27 & E93 & 82 & $\ldots$ & $\ldots$ & 101 & 137 \\
\hline $\mathrm{Ca} I$ & 6166.44 & 2.52 & -1.14 & R03 & 95 & 80 & 118 & 101 & 128 \\
\hline $\mathrm{Ca}$ I & 6169.04 & 2.52 & -0.80 & R03 & $\ldots$ & $\ldots$ & $\ldots$ & 114 & $\ldots$ \\
\hline $\mathrm{Ca} I$ & 6169.56 & 2.53 & -0.48 & DS91 & $\ldots$ & $\ldots$ & $\ldots$ & 142 & $\ldots$ \\
\hline $\mathrm{Ca} I$ & 6455.60 & 2.51 & -1.29 & R03 & $\ldots$ & 80 & 101 & 90 & $\ldots$ \\
\hline $\mathrm{Ca}$ I & 6471.66 & 2.51 & -0.69 & S86 & 122 & 114 & $\ldots$ & $\ldots$ & $\ldots$ \\
\hline $\mathrm{Ca} I$ & 6493.79 & 2.52 & -0.11 & DS91 & 144 & $\ldots$ & $\ldots$ & $\ldots$ & $\ldots$ \\
\hline Ti I & 4759.28 & 2.25 & 0.514 & MFK & $\cdots$ & 86 & $\cdots$ & 88 & $\cdots$ \\
\hline Ti I & 4820.41 & 1.50 & -0.439 & MFK & $\cdots$ & 65 & $\ldots$ & 92 & $\cdots$ \\
\hline Ti I & 5009.66 & 0.02 & -2.259 & MFK & $\ldots$ & $\ldots$ & 112 & 88 & $\ldots$ \\
\hline Ti I & 5039.96 & 0.02 & -1.130 & MFK & $\ldots$ & 123 & $\ldots$ & $\ldots$ & $\ldots$ \\
\hline Ti I & 5043.59 & 0.84 & -1.733 & MFK & $\ldots$ & $\ldots$ & 73 & $\ldots$ & 111 \\
\hline Ti I & 5062.10 & 2.16 & -0.464 & MFK & 35 & $\ldots$ & $\ldots$ & $\ldots$ & 64 \\
\hline $\mathrm{Ti}$ I & 5113.45 & 1.44 & -0.880 & E93 & $\ldots$ & $\ldots$ & $\ldots$ & $\ldots$ & 106 \\
\hline Ti I & 5147.48 & 0.00 & -2.012 & MFK & $\ldots$ & 89 & $\ldots$ & $\ldots$ & $\ldots$ \\
\hline Ti I & 5210.39 & 0.05 & -0.883 & MFK & $\ldots$ & $\ldots$ & $\ldots$ & 156 & $\ldots$ \\
\hline Ti I & 5219.71 & 0.02 & -2.292 & MFK & $\ldots$ & $\ldots$ & 117 & $\ldots$ & $\ldots$ \\
\hline Ti I & 5295.78 & 1.05 & -1.633 & MFK & $\ldots$ & 36 & $\ldots$ & 56 & 64 \\
\hline Ti I & 5689.48 & 2.30 & -0.469 & MFK & $\ldots$ & $\ldots$ & $\ldots$ & 38 & 67 \\
\hline Ti I & 5866.46 & 1.07 & -0.871 & E93 & $\cdots$ & 91 & $\cdots$ & $\ldots$ & 155 \\
\hline Ti I & 5922.12 & 1.05 & -1.465 & MFK & $\ldots$ & $\ldots$ & $\ldots$ & $\ldots$ & 101 \\
\hline Ti I & 5978.55 & 1.87 & -0.496 & MFK & $\ldots$ & 43 & $\ldots$ & 76 & $\ldots$ \\
\hline Ti I & 6126.22 & 1.07 & -1.370 & R03 & $\ldots$ & 44 & $\ldots$ & 71 & $\ldots$ \\
\hline Ti I & 6258.11 & 1.44 & -0.355 & MFK & $\ldots$ & 96 & $\ldots$ & $\ldots$ & 149 \\
\hline Ti I & 6261.11 & 1.43 & -0.480 & B86 & 95 & 86 & 124 & 105 & $\ldots$ \\
\hline Ti I & 6554.24 & 1.44 & -1.219 & MFK & $\ldots$ & $\ldots$ & $\ldots$ & 51 & 84 \\
\hline $\mathrm{Cr}_{\mathrm{I}}$ & 4836.85 & 3.10 & -1.137 & MFK & $\ldots$ & $\ldots$ & 49 & $\ldots$ & $\ldots$ \\
\hline Cr I & 5193.50 & 3.42 & -0.720 & MFK & $\ldots$ & $\ldots$ & $\ldots$ & 23 & $\ldots$ \\
\hline $\mathrm{Cr}_{\mathrm{I}}$ & 5196.45 & 3.45 & -0.270 & MFK & $\ldots$ & $\ldots$ & 92 & $\ldots$ & $\ldots$ \\
\hline $\mathrm{Cr}_{\mathrm{I}}$ & 5214.13 & 3.37 & -0.740 & MFK & $\ldots$ & $\ldots$ & $\ldots$ & 32 & $\ldots$ \\
\hline Cr I & 5296.70 & 0.98 & -1.390 & GS & $\ldots$ & 129 & $\ldots$ & $\ldots$ & $\ldots$ \\
\hline $\mathrm{Cr}$ I & 5348.32 & 1.00 & -1.290 & GS & $\cdots$ & 126 & $\cdots$ & 154 & $\cdots$ \\
\hline Cr I & 5702.32 & 3.45 & -0.666 & MFK & 21 & $\ldots$ & 63 & $\ldots$ & $\ldots$ \\
\hline $\mathrm{Cr}_{\mathrm{I}}$ & 5783.07 & 3.32 & -0.500 & MFK & $\ldots$ & $\ldots$ & $\ldots$ & 54 & 75 \\
\hline $\mathrm{Cr}_{\mathrm{I}}$ & 5783.87 & 3.32 & -0.290 & GS & 49 & 60 & 95 & 57 & 90 \\
\hline $\mathrm{Cr}_{\mathrm{I}}$ & 5787.92 & 3.32 & -0.080 & GS & 51 & $\ldots$ & 100 & $\ldots$ & 103 \\
\hline $\mathrm{Cr}_{\mathrm{I}}$ & 6330.09 & 0.94 & -2.920 & R03 & $\ldots$ & 67 & $\ldots$ & $\ldots$ & $\ldots$ \\
\hline $\mathrm{Ni}$ I & 4904.42 & 3.54 & -0.170 & MFK & 117 & $\ldots$ & 139 & $\ldots$ & $\ldots$ \\
\hline $\mathrm{Ni}$ I & 4935.83 & 3.94 & -0.360 & MFK & $\ldots$ & $\cdots$ & 99 & 78 & $\ldots$ \\
\hline $\mathrm{Ni}$ I & 4953.21 & 3.74 & -0.660 & MFK & 61 & 65 & $\ldots$ & $\ldots$ & 105 \\
\hline $\mathrm{Ni}$ I & 4967.52 & 3.80 & -1.570 & MFK & $\ldots$ & $\ldots$ & $\ldots$ & 35 & $\ldots$ \\
\hline $\mathrm{Ni}$ I & 5010.94 & 3.63 & -0.870 & MFK & $\ldots$ & $\cdots$ & 78 & 68 & $\ldots$ \\
\hline
\end{tabular}


Table 8

(Continued)

\begin{tabular}{|c|c|c|c|c|c|c|c|c|c|}
\hline \multirow[b]{3}{*}{ Element } & \multirow[b]{3}{*}{$\lambda$} & \multirow[b]{3}{*}{$\chi(\mathrm{eV})$} & \multirow[b]{3}{*}{$\log g f$} & \multirow[b]{3}{*}{ References } & \multicolumn{5}{|c|}{ 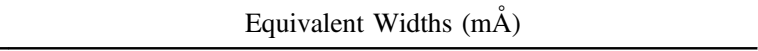 } \\
\hline & & & & & \multicolumn{5}{|c|}{ Star } \\
\hline & & & & & 1534 & 1616 & 1349 & 784 & 663 \\
\hline Ni I & 5048.85 & 3.85 & -0.370 & MFK & 94 & $\cdots$ & $\cdots$ & $\cdots$ & $\cdots$ \\
\hline Ni I & 5084.11 & 3.68 & -0.180 & E93 & $\cdots$ & 94 & $\cdots$ & $\cdots$ & $\cdots$ \\
\hline $\mathrm{Ni}$ I & 5094.42 & 3.83 & -1.080 & MFK & 51 & 53 & $\cdots$ & $\cdots$ & $\cdots$ \\
\hline $\mathrm{Ni}$ I & 5115.40 & 3.83 & -0.280 & R03 & $\ldots$ & 95 & $\ldots$ & $\ldots$ & $\ldots$ \\
\hline Ni I & 5157.98 & 3.61 & -1.590 & MFK & $\cdots$ & $\cdots$ & 44 & $\cdots$ & $\cdots$ \\
\hline $\mathrm{Ni}$ I & 5578.73 & 1.68 & -2.640 & MFK & $\cdots$ & $\cdots$ & $\ldots$ & 105 & $\cdots$ \\
\hline Ni I & 5589.37 & 3.90 & -1.140 & MFK & $\cdots$ & $\cdots$ & 46 & $\cdots$ & $\cdots$ \\
\hline Ni I & 5593.75 & 3.90 & -0.840 & MFK & $\cdots$ & 51 & 68 & $\cdots$ & 84 \\
\hline $\mathrm{Ni}$ I & 5643.09 & 4.17 & -1.250 & MFK & $\cdots$ & $\cdots$ & 27 & $\ldots$ & $\ldots$ \\
\hline $\mathrm{Ni}$ I & 5748.36 & 1.68 & -3.260 & MFK & $\ldots$ & 64 & $\ldots$ & $\ldots$ & 113 \\
\hline $\mathrm{Ni}$ I & 5760.84 & 4.11 & -0.800 & MFK & $\ldots$ & 59 & 61 & $\ldots$ & $\ldots$ \\
\hline $\mathrm{Ni}$ I & 5805.23 & 4.17 & -0.640 & MFK & $\ldots$ & $\ldots$ & $\ldots$ & $\ldots$ & 59 \\
\hline $\mathrm{Ni}$ I & 5996.74 & 4.24 & -1.060 & MFK & $\ldots$ & $\ldots$ & $\ldots$ & $\ldots$ & 57 \\
\hline $\mathrm{Ni}$ I & 6086.29 & 4.27 & -0.510 & MFK & $\ldots$ & $\cdots$ & $\ldots$ & 77 & $\ldots$ \\
\hline $\mathrm{Ni}$ I & 6108.12 & 1.68 & -2.440 & MFK & 124 & 90 & 140 & $\ldots$ & $\ldots$ \\
\hline Ni I & 6111.08 & 4.09 & -0.870 & MFK & $\ldots$ & $\cdots$ & $\ldots$ & $\cdots$ & 75 \\
\hline $\mathrm{Ni}$ I & 6128.98 & 1.68 & -3.320 & MFK & $\cdots$ & 48 & $\ldots$ & 69 & 105 \\
\hline $\mathrm{Ni}$ I & 6176.82 & 4.09 & -0.264 & R03 & $\ldots$ & $\ldots$ & $\cdots$ & 86 & 114 \\
\hline Ni I & 6177.25 & 1.83 & -3.510 & MFK & $\cdots$ & $\cdots$ & $\ldots$ & 45 & 67 \\
\hline $\mathrm{Ni}$ I & 6186.72 & 4.11 & -0.960 & MFK & 38 & $\cdots$ & $\ldots$ & 46 & $\ldots$ \\
\hline $\mathrm{Ni}$ I & 6204.61 & 4.09 & -1.150 & MFK & $\ldots$ & 34 & $\ldots$ & 49 & 56 \\
\hline $\mathrm{Ni}$ I & 6223.99 & 4.11 & -0.980 & MFK & $\ldots$ & $\ldots$ & $\ldots$ & 50 & $\ldots$ \\
\hline $\mathrm{Ni}$ I & 6230.10 & 4.11 & -1.260 & MFK & $\ldots$ & 27 & $\ldots$ & $\ldots$ & $\ldots$ \\
\hline $\mathrm{Ni}$ I & 6327.60 & 1.68 & -3.150 & MFK & 60 & 74 & $\ldots$ & 93 & 99 \\
\hline $\mathrm{Ni}$ I & 6482.81 & 1.94 & -2.630 & MFK & $\cdots$ & 82 & 112 & 96 & $\cdots$ \\
\hline $\mathrm{Ni}$ I & 6586.32 & 1.95 & -2.810 & MFK & 76 & $\ldots$ & 92 & 76 & $\ldots$ \\
\hline $\mathrm{Ni}$ I & 6635.14 & 4.42 & -0.830 & MFK & $\ldots$ & $\ldots$ & $\ldots$ & 31 & 43 \\
\hline $\mathrm{Ni}$ I & 6643.64 & 1.68 & -2.030 & MFK & $\ldots$ & 129 & $\ldots$ & 139 & $\ldots$ \\
\hline $\mathrm{Ni}$ I & 6767.78 & 1.83 & -2.170 & MFK & 131 & $\ldots$ & $\ldots$ & $\ldots$ & 155 \\
\hline $\mathrm{Ni}$ I & 6772.32 & 3.66 & -0.970 & $\mathrm{R} 03$ & $\ldots$ & $\ldots$ & $\cdots$ & 91 & 90 \\
\hline $\mathrm{Ni}$ I & 7788.93 & 1.95 & -1.990 & E93 & $\ldots$ & 118 & 156 & 142 & $\ldots$ \\
\hline
\end{tabular}

References. B86: Blackwell et al. (1986), Ca07: Carretta et al. (2007), D2002: Depagne et al. (2002), DS91: Drake \& Smith (1991), E93: Edvardsson et al. (1993), GS: Gratton \& Sneden (1988), MFK: Martin et al. (2002), MR94: McWilliam \& Rich (1994), PS: Preston \& Sneden (2001), R03: Reddy et al. (2003), R99: Reddy et al. (1999), WSM: Wiese et al. (1969).

Table 9

Atmospheric Parameters from Photometry (ph) and Spectroscopy (sp)

\begin{tabular}{lcccccrcc}
\hline \hline ID & $\begin{array}{c}T_{\text {eff,ph }} \\
(\mathrm{K})\end{array}$ & $\begin{array}{c}\log g_{\text {ph }} \\
(\mathrm{dex})\end{array}$ & $\begin{array}{c}T_{\text {eff, sp }} \\
(\mathrm{K})\end{array}$ & $\begin{array}{c}\log g_{s p} \\
(\mathrm{dex})\end{array}$ & $\begin{array}{c}\xi \\
\left(\mathrm{km} \mathrm{s}^{-1}\right)\end{array}$ & {$[\mathrm{Fe} \mathrm{I} / \mathrm{H}] \pm \sigma(\#)$} & {$[\mathrm{Fe}$ II $/ \mathrm{H}] \pm \sigma(\#)$} & Comment \\
\hline 395 & 5205 & 2.74 & 5100 & 2.7 & 1.6 & $-0.15 \pm 0.15(53)$ & $-0.15 \pm 0.12(5)$ & Member \\
663 & 5123 & 2.85 & 4900 & 3.2 & 2.3 & $0.07 \pm 0.14(37)$ & $0.06 \pm 0.13(3)$ & Member \\
769 & 5196 & 2.53 & 5200 & 3.0 & 1.3 & $0.10 \pm 0.14(53)$ & $0.08 \pm 0.17(3)$ & Member \\
784 & 4955 & 2.82 & 5000 & 2.5 & 1.7 & $-0.08 \pm 0.13(39)$ & $-0.11 \pm 0.12(6)$ & Non-Member \\
806 & 6324 & 2.86 & 6000 & 2.7 & 4.9 & $-0.53 \pm 0.12(20)$ & $-0.52(2)$ & Non-Member $/$ binary Cepheid? \\
1110 & 5161 & 2.68 & 5350 & 3.4 & 1.0 & $0.03 \pm 0.15(44)$ & $0.01 \pm 0.09(6)$ & Member \\
1118 & 4958 & 2.63 & 5100 & 2.6 & 1.5 & $-0.16 \pm 0.12(44)$ & $-0.17 \pm 0.10(3)$ & Member \\
1349 & 4796 & 2.72 & 5100 & 2.6 & 2.1 & $0.01 \pm 0.19(25)$ & $0.01(2)$ & Member \\
1534 & 4982 & 2.73 & 5000 & 2.0 & 2.2 & $-0.30 \pm 0.16(15)$ & $-0.29(2)$ & Non-Member \\
1616 & 5465 & 2.83 & 5450 & 3.5 & 1.7 & $-0.07 \pm 0.16(31)$ & $-0.09 \pm 0.14(5)$ & Non-Member \\
\hline
\end{tabular}

Note. For $[\mathrm{Fe} \mathrm{I} / \mathrm{H}]$ and $[\mathrm{Fe}$ II $/ \mathrm{H}]$, we also show the standard deviation and the number of lines employed.

ratios of $0.17,0.24,0.03$, and 0.13 , respectively (Andrievsky et al. 2014). The chemical abundances of $\mathrm{Ba}$ in disk Cepheids is known to suffer from NLTE effects (Andrievsky et al. 2013; Andrievsky et al. 2014). However, the NLTE correction for the Ba II line $5853 \AA$ is not especially large, averaging around -0.1 dex (Andrievsky et al. 2013), and does not have any significant effect on the high overabundance obtained for $\mathrm{XZ}$ CMa. We will discuss the case of this star in the final section.

\section{DISCUSSION AND CONCLUSIONS}

In this paper we have presented the first study of Tombaugh 1 using both high-resolution spectroscopy and precision 
Table 10

Adopted Solar Abundances

\begin{tabular}{lccc}
\hline \hline Element & This Work & Grevesse $\&$ Sauval (1998) & Asplund et al. (2009) \\
\hline $\mathrm{Fe}$ & 7.50 & 7.50 & 7.50 \\
$\mathrm{Na}$ & 6.26 & 6.33 & 6.24 \\
$\mathrm{Mg}$ & 7.55 & 7.58 & 7.60 \\
$\mathrm{Al}$ & 6.31 & 6.47 & 6.45 \\
$\mathrm{Si}$ & 7.61 & 7.55 & 7.51 \\
$\mathrm{Ca}$ & 6.37 & 6.36 & 6.34 \\
$\mathrm{Ti}$ & 4.93 & 5.02 & 4.95 \\
$\mathrm{Cr}$ & 5.65 & 5.67 & 5.64 \\
$\mathrm{Ni}$ & 6.29 & 6.25 & 6.22 \\
$\mathrm{Y}$ & 2.04 & 2.24 & 2.21 \\
$\mathrm{Ba}$ & 2.18 & 2.13 & 2.18 \\
$\mathrm{Ce}$ & 1.48 & 1.58 & 1.58 \\
$\mathrm{Nd}$ & 1.42 & 1.50 & 1.42 \\
\hline
\end{tabular}

$u v b y \mathrm{CaH} \beta$ photometry. Our results for the abundance ratios of elements from $\mathrm{Na}$ to $\mathrm{Ni}$ and the cluster fundamental parameters of distance and age tag this open cluster as an intermediate-age $(0.95 \mathrm{Gyr})$ cluster belonging to the galactic thin disk. As such, it allows the addition of one more data point to the census of star clusters used to map the chemical history of the disk, falling within a galactocentric zone where there is universal agreement that a significant change in mean metallicity occurs among all classes of objects populating the thin disk. Where disagreement arises is in the exact form and location of the transition region. Does Tombaugh 1 lie along a uniform linear gradient extending from $R_{\mathrm{GC}}=5$ to $20 \mathrm{kpc}$, or does the gradient change slope beyond the solar circle? If it changes, where does the transition occur and why? The growing evidence from studies of distant anticenter open clusters and Cepheids (e.g., Magrini et al. 2009; Lépine et al. 2011; Yong et al. 2012; Korotin et al. 2014, among others) is that the metallicity gradient beyond $R_{\mathrm{GC}}=13 \mathrm{kpc}$ is considerably flatter than that between 9 and $13 \mathrm{kpc}$ (see Figure 15).

In Figure 15 we show the radial metallicity gradient from Magrini et al. (2009) (blue squares), with the addition of our spectroscopic results for Tombaugh 1 (red circle) with [Fe/ $\mathrm{H}]=-0.02 \pm 0.05$ and $R_{\mathrm{GC}}=10.46 \mathrm{kpc}$. If we use the lower photometric value of $[\mathrm{Fe} / \mathrm{H}]=-0.10, R_{\mathrm{GC}}$ would be reduced to $10.36 \mathrm{kpc}$, a negligible shift in distance on this scale. Also plotted are additional open clusters analyzed with highresolution spectroscopy (green squares): IC 4725 and NGC 6087 (Gratton 2000); NGC 6603, NGC 2539, NGC 2447, IC 2714, and NGC 5822 (Santos et al. 2009); NGC 6192, NGC 6404, and NGC 6583 (Magrini et al. 2010); NGC 7160 (Monroe \& Pilachowski 2010); Cr 110, NGC 2099, NGC 2420, and NGC 7789 (Pancino et al. 2010); Tombaugh 2 (Villanova et al. 2010); NGC 3114 (Santrich et al. 2013); NGC 4815 and NGC 6705 (Magrini et al. 2014); NGC 4337 (Carraro et al. 2014b); and Trumpler 20 (Carraro et al. 2014c). The use of the spectroscopic value alone is tied to an apparent offset between the photometric abundance scale, for Strömgren photometry tied to high-dispersion spectroscopy of F dwarfs, and the red giant high-dispersion spectroscopic scale, often distantly coupled to the Sun. The issue is apparent in Figure 15 where, inside $R_{\mathrm{GC}}=9.8 \mathrm{kpc}$, no cluster has $[\mathrm{Fe} / \mathrm{H}]$ below -0.1 and, more important, even ignoring the super-metal-rich outliers, the typical cluster $[\mathrm{Fe} / \mathrm{H}]$ at all ages is +0.1 . While a virtually identical pattern was found by Twarog et al. (1997), the lower limit and mean abundances from photometry and medium-resolution spectroscopy of cluster red giants were [Fe/ $\mathrm{H}]=-0.2$ and 0.0 , respectively. Similar offsets between spectroscopic abundances of red giants and the photometry of $\mathrm{F}$ dwarfs have been found in NGC 3680 (Anthony-Twarog et al. 2009), NGC 5822 (Carraro et al. 2011), NGC 6819 (Anthony-Twarog et al. 2014), and NGC 752 (Twarog et al. 2015). In the cases of NGC 3680, NGC 6819, and NGC 752, high-dispersion spectroscopic analysis of the $\mathrm{F}$ dwarfs agrees with the photometric abundances. If this offset to the spectroscopic scale applies to giants across all metallicities, the trend in Figure 15 remains correct, even if the curve is shifted vertically by 0.1 dex.

We observe that Tombaugh 1 is consistent with the trend defined by Magrini et al. (2009) for the metallicity gradient, with Tombaugh 1 located in the inner disk $\left(R_{\mathrm{GC}} \lesssim 12 \mathrm{kpc}\right)$. The existence of an apparent transition zone ranging from $R_{\mathrm{GC}}=10$ to $12 \mathrm{kpc}$ between an inner and outer disk lends support to the contention that the metallicity evolution in these two regions occurs in different ways (Magrini et al. 2009; Lépine et al. 2011). According to Lépine et al. (2011), this behavior is due to a barrier created by a void in the interstellar gas in the region of the corotation radius of the main spiral structure. This dynamical interaction produces an inward flow of the gas on the inside of the corotation zone of the Galaxy but an outward flow in the outer disk regions.

In recent years, the abundances of the $s$-process elements in open clusters have become a target of intense study (e.g., D'Orazi et al. 2009, 2012; Jacobson et al. 2011; Maiorca et al. 2011; Jacobson \& Friel 2013; Mishenina et al. 2013, 2015). This recent interest was sparked by the unexpected results of D'Orazi et al. (2009) for a sample of 20 open clusters. D'Orazi et al. (2009) found that $[\mathrm{Ba} / \mathrm{Fe}]$ increases as cluster age decreases, contrary to the predictions of yields for $\mathrm{Ba}$ from AGB stars (e.g., Travaglio et al. 1999; Busso et al. 2001). Later work supplied confirmation for other $s$-process elements from unevolved stars in open clusters: Ba (Jacobson \& Friel 2013; Mishenina et al. 2013); Ba and La (Jacobson et al. 2011); and Y, Zr, La, and Ce (Maiorca et al. 2011). However, Jacobson \& Friel (2013) did not find a trend for [X/Fe] for $\mathrm{La}$ and $\mathrm{Zr}$ versus age for their sample of 19 open clusters, which could indicate that the source of the $s$-process abundance trend with age does not affect all $s$-process elements equally. Among field stars, some s-process elements, $\mathrm{Zr}$ (Reddy et al. 2003) and $\mathrm{Ba}$ (Bensby et al. 2005), also show an increase in $[\mathrm{X} / \mathrm{Fe}]$ with decreasing age, while others, Y (Bensby et al. 2005), Ba, and Ce (Reddy et al. 2003), do not.

In this context, our photometric and spectroscopic analysis classifies Tombaugh 1 as having intermediate age (0.95 Gyr), with an enrichment of heavy $s$-process elements (Ba with $+0.35 \pm 0.03 \mathrm{dex}$, Ce with $+0.25 \pm 0.06 \mathrm{dex}$, and $\mathrm{Nd}$ with $+0.37 \pm 0.05 \mathrm{dex})$ and solar values to $\mathrm{Y}(+0.06 \pm 0.04)$, indicating a high efficiency in the synthesis of the $s$-process elements. Some open clusters with similar ages show enrichment of the $s$-process elements similar to that found for Tombaugh 1, e.g., NGC 5822 (0.9 Gyr) (Carraro et al. 2011) and NGC 3680 (1.7 Gyr) (Anthony-Twarog et al. 2009) with $[\mathrm{Ce} / \mathrm{Fe}]=0.25$ and $[\mathrm{Ce} / \mathrm{Fe}]=0.26$, respectively (Maiorca et al. 2011).

The reason why open clusters younger than $\sim 1.5 \mathrm{Gyr}$ (Maiorca et al. 2011) contain an overabundance of some $s$ process elements (mainly $\mathrm{Ba}$ ) compared to the old open clusters still is not understood. D'Orazi et al. (2009) and Maiorca et al. 
Table 11

Abundance Ratios ([X/Fe]) for the Elements from $\mathrm{Na}$ to $\mathrm{Cr}$ for the Stars Observed

\begin{tabular}{|c|c|c|c|c|c|c|c|}
\hline \multicolumn{8}{|c|}{ Cluster Giants } \\
\hline ID & {$[\mathrm{Na} / \mathrm{Fe}] \mathrm{NLTE}$} & {$[\mathrm{Mg} / \mathrm{Fe}]$} & {$[\mathrm{Al} / \mathrm{Fe}]$} & {$[\mathrm{Si} / \mathrm{Fe}]$} & {$[\mathrm{Ca} / \mathrm{Fe}]$} & {$[\mathrm{Ti} / \mathrm{Fe}]$} & {$[\mathrm{Cr} / \mathrm{Fe}]$} \\
\hline 395 & $+0.15(2)$ & $+0.10 \pm 0.13(3)$ & $\ldots$ & $+0.13 \pm 0.09(4)$ & $+0.02 \pm 0.13(7)$ & $+0.07(1)$ & $+0.02 \pm 0.14(7)$ \\
\hline 663 & $+0.12(2)$ & $+0.03 \pm 0.12(3)$ & $+0.15 \pm 0.04(3)$ & $+0.23 \pm 0.11(4)$ & $-0.06(2)$ & $+0.04 \pm 0.14(9)$ & $-0.10 \pm 0.02(3)$ \\
\hline 769 & $+0.04(2)$ & $-0.12 \pm 0.13(4)$ & $\ldots$ & $-0.05 \pm 0.04(5)$ & $-0.05 \pm 0.15(7)$ & $+0.10 \pm 0.11(18)$ & $+0.04 \pm 0.12(7)$ \\
\hline 1110 & $+0.13(2)$ & $+0.03 \pm 0.14(3)$ & $\cdots$ & $-0.14(2)$ & $+0.04 \pm 0.11(5)$ & $+0.09 \pm 0.13(11)$ & $+0.18 \pm 0.06(6)$ \\
\hline 1118 & $+0.38(2)$ & $+0.23 \pm 0.07(4)$ & $\ldots$ & $+0.07 \pm 0.09(3)$ & $+0.12 \pm 0.08(5)$ & $+0.08 \pm 0.13(13)$ & $+0.14 \pm 0.13(6)$ \\
\hline 1349 & $\ldots$ & $-0.11(2)$ & $+0.16(2)$ & $-0.19(2)$ & $-0.01(2)$ & $+0.28 \pm 0.11(4)$ & $+0.30 \pm 0.09(5)$ \\
\hline Tombaugh $1^{\mathrm{a}}$ & $+0.17 \pm 0.06$ & $+0.03 \pm 0.05$ & +0.15 & $+0.01 \pm 0.07$ & $+0.01 \pm 0.03$ & $+0.11 \pm 0.04$ & $+0.10 \pm 0.06$ \\
\hline
\end{tabular}

Field Stars

\begin{tabular}{lccccccc}
\hline ID & {$[\mathrm{Na} / \mathrm{Fe}] N L T E$} & {$[\mathrm{Mg} / \mathrm{Fe}]$} & {$[\mathrm{Al} / \mathrm{Fe}]$} & {$[\mathrm{Si} / \mathrm{Fe}]$} & {$[\mathrm{Ca} / \mathrm{Fe}]$} & {$[\mathrm{Ti} / \mathrm{Fe}]$} & {$[\mathrm{Cr} / \mathrm{Fe}]$} \\
\hline 784 & $+0.20(2)$ & $+0.17 \pm 0.13(4)$ & $+0.27(2)$ & $+0.14 \pm 0.10(5)$ & $-0.06 \pm 0.09(6)$ & $+0.03 \pm 0.07(10)$ & $-0.13 \pm 0.10(5)$ \\
$806^{\mathrm{b}}$ & $+0.27(1)$ & $+0.02(1)$ & $\ldots$ & $+0.14 \pm 0.11(4)$ & $+0.05(2)$ & $+0.32(1)$ & $-0.12 \pm 0.08(3)$ \\
1534 & $+0.21(1)$ & $+0.25 \pm 0.02(3)$ & $\ldots$ & $-0.15 \pm 0.09(5)$ & $-0.20 \pm 0.15(4)$ & $+0.01(2)$ & $-0.22 \pm 0.11(3)$ \\
1616 & $+0.34(1)$ & $+0.18 \pm 0.03(3)$ & $-0.10(2)$ & $-0.21 \pm 0.08(3)$ & $-0.01 \pm 0.06(3)$ & $+0.27 \pm 0.13(10)$ & $+0.17 \pm 0.14(4)$ \\
\hline
\end{tabular}

Notes. For all abundance ratios, we also show the standard deviation and the number of lines employed. $[\mathrm{Na} / \mathrm{Fe}]$ accounts for the NLTE effects calculated as in Gratton et al. (1999); see text.

a Mean abundance ratio for each element for Tombaugh 1.

b The classical Cepheid XZ CMa.

Table 12

Abundance Ratios ([X/Fe]) for the Elements from Ni to Nd for the Stars Observed

\begin{tabular}{|c|c|c|c|c|c|}
\hline \multicolumn{6}{|c|}{ Cluster Giants } \\
\hline ID & {$[\mathrm{Ni} / \mathrm{Fe}]$} & {$[\mathrm{Y} / \mathrm{Fe}]$} & {$[\mathrm{Ba} / \mathrm{Fe}]$} & {$[\mathrm{Ce} / \mathrm{Fe}]$} & {$[\mathrm{Nd} / \mathrm{Fe}]$} \\
\hline 395 & $-0.06 \pm 0.13(18)$ & $-0.10(1)$ & $+0.28(1)$ & $+0.25(1)$ & $+0.35(1)$ \\
\hline 663 & $-0.04 \pm 0.15(14)$ & $+0.00(1)$ & $\ldots$ & $\ldots$ & $\ldots$ \\
\hline 769 & $+0.02 \pm 0.15(28)$ & $+0.07(1)$ & $+0.38(1)$ & $+0.10(1)$ & $+0.30(1)$ \\
\hline 1110 & $-0.05 \pm 0.13(19)$ & $+0.14(1)$ & $+0.37(1)$ & $+0.37(1)$ & $+0.47(1)$ \\
\hline 1118 & $+0.04 \pm 0.12(19)$ & $+0.13(1)$ & $+0.36(1)$ & $+0.29(1)$ & $\ldots$ \\
\hline 1349 & $-0.14 \pm 0.09(12)$ & $\ldots$ & $\ldots$ & $\ldots$ & $\cdots$ \\
\hline Tombaugh $1^{\mathrm{a}}$ & $-0.04 \pm 0.02$ & $+0.06 \pm 0.04$ & $+0.35 \pm 0.03$ & $+0.25 \pm 0.06$ & $+0.37 \pm 0.05$ \\
\hline \multicolumn{6}{|c|}{ Field Stars } \\
\hline ID & {$[\mathrm{Ni} / \mathrm{Fe}]$} & {$[\mathrm{Y} / \mathrm{Fe}]$} & {$[\mathrm{Ba} / \mathrm{Fe}]$} & {$[\mathrm{Ce} / \mathrm{Fe}]$} & {$[\mathrm{Nd} / \mathrm{Fe}]$} \\
\hline 784 & $-0.14 \pm 0.14(18)$ & $+0.30(1)$ & $-0.02(1)$ & $+0.20(1)$ & $+0.32(1)$ \\
\hline $806^{\mathrm{b}}$ & $+0.20 \pm 0.16(3)$ & $\ldots$ & $+0.73(1)$ & $\ldots$ & $\ldots$ \\
\hline 1534 & $-0.27 \pm 0.13(9)$ & $-0.07(1)$ & $-0.05(1)$ & $\cdots$ & $\ldots$ \\
\hline 1616 & $+0.02 \pm 0.13(15)$ & $-0.11(1)$ & $+0.39(1)$ & $\cdots$ & $+0.04(1)$ \\
\hline
\end{tabular}

Notes. For all abundance ratios, we also show the standard deviation and the number of lines employed.

a Mean abundance ratio for each element for Tombaugh 1.

b The classical Cepheid XZ CMa.

(2011) have proposed a scenario with models of extra-mixing phenomena with high efficiency in the production of the neutron source ${ }^{13} \mathrm{C}$ in stars with $M \leqslant 1.5 M_{\odot}$ (Busso et al. 2007; Nordhaus et al. 2008; Nucci \& Busso 2014; Trippella et al. 2014). Very recently, Mishenina et al. (2015) suggested that the Ba overabundance in open clusters could be due to action from the intermediate neutron-capture process, or $i$ process (Cowan \& Rose 1977). However, as Mishenina et al. (2015) pointed out, it remains difficult to know which open cluster stars would be the host of the $i$ process; low-metallicity stars are a more probable example of these hosts (Bertolli et al. 2013; Dardelet et al. 2015). Indeed, confirmation of the enrichment of $s$-process elements in young clusters requires the analysis of a large and homogeneous sample of young and old open clusters with well-determined $s$-process abundances.

The low number of open clusters with both reliable photometric and spectroscopic parameters, about $13.2 \%$ of the known open clusters as defined by the 2014 update of the Dias et al. (2002) catalog, is just one of the factors that hinder a definitive characterization of the galactic metallicity gradient, as well as its variation over time and azimuthally within the disk for individual elements. Studies of other poorly known open clusters like Tombaugh 1 using high-resolution spectroscopy and precision photometry to define reliably all of the key 
Table 13

Abundance Uncertainties for Star 769

\begin{tabular}{lcccc}
\hline \hline Element & $\begin{array}{c}\Delta T_{\text {eff }} \\
+180 \mathrm{~K}\end{array}$ & $\begin{array}{c}\Delta \log g \\
+0.3\end{array}$ & $\begin{array}{c}\Delta \xi \\
+0.3 \mathrm{~km} \mathrm{~s}^{-1}\end{array}$ & $\left(\sum \sigma^{2}\right)^{1 / 2}$ \\
\hline $\mathrm{Fe}_{\text {I }}$ & +0.14 & 0.00 & -0.14 & 0.20 \\
$\mathrm{Fe}$ II & -0.11 & +0.19 & -0.11 & 0.25 \\
$\mathrm{Na}_{\text {I }}$ & +0.12 & -0.01 & -0.05 & 0.13 \\
$\mathrm{Mg}_{\text {I }}$ & +0.09 & -0.01 & -0.07 & 0.11 \\
$\mathrm{Al}_{\text {I }}$ & +0.09 & -0.04 & -0.05 & 0.11 \\
$\mathrm{Si}_{\text {I }}$ & 0.00 & +0.05 & -0.05 & 0.07 \\
$\mathrm{Ca}_{\text {I }}$ & +0.16 & -0.04 & -0.15 & 0.22 \\
$\mathrm{Ti}_{\text {I }}$ & +0.23 & -0.02 & -0.12 & 0.26 \\
$\mathrm{Cr}_{\text {I }}$ & +0.15 & -0.02 & -0.09 & 0.18 \\
$\mathrm{Ni}_{\text {I }}$ & +0.11 & +0.03 & -0.12 & 0.17 \\
$\mathrm{Y}_{\text {II }}$ & -0.02 & +0.08 & -0.05 & 0.10 \\
$\mathrm{Zr}_{\text {I }}$ & +0.01 & -0.03 & -0.06 & 0.07 \\
$\mathrm{Ba}_{\text {II }}$ & +0.12 & +0.17 & -0.14 & 0.25 \\
$\mathrm{Ce}_{\text {II }}$ & -0.02 & +0.05 & -0.10 & 0.11 \\
$\mathrm{Nd}_{\text {II }}$ & +0.05 & +0.15 & -0.05 & 0.17 \\
\hline
\end{tabular}

Note. Each column gives the variation of the abundance caused by the variation in $T_{\text {eff }}, \log g$, and $\xi$. The last column gives the compounded rms uncertainty of the second to fourth columns. The abundance uncertainties of aluminum were calculated using the star 663 .

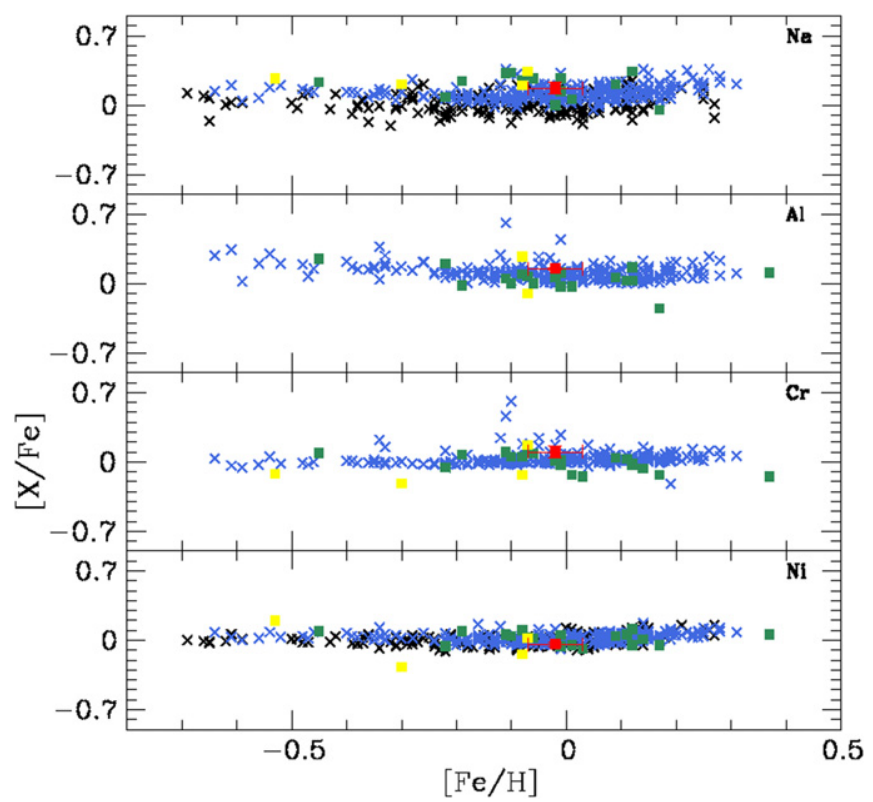

Figure 11. Abundance ratios $[\mathrm{X} / \mathrm{Fe}]$ vs. $[\mathrm{Fe} / \mathrm{H}]$. Blue crosses: field giants of Luck \& Heiter (2007); black crosses: clump giants of Mishenina et al. (2006); yellow squares: our sample of field giant stars; red squares: our mean abundances of Tombaugh 1; green squares: open clusters from the literature (NGC 6192, NGC 6404, and NGC 6583 of Magrini et al. 2010; NGC 3114 of Santrich et al. 2013; NGC 2527, NGC 2682, NGC 2482, NGC 2539, NGC 2335, NGC 2251, and NGC 2266 of Reddy et al. 2013; NGC 4337 of Carraro et al. 2014b; Trumpler 20 of Carraro et al. 2014c; NGC 4815 and NGC 6705 of Magrini et al. 2014; Cr 110, Cr 261, NGC 2477, NGC 2506, and NGC 5822 of Mishenina et al. 2015).

parameters that influence plots like Figures $11-13$ and 15 remain the key to forward progress in disentangling the complex system known as the galactic disk. The next step in this direction is being conducted by large surveys like GaiaESO mapping the chemistry of all of the components of the Galaxy.

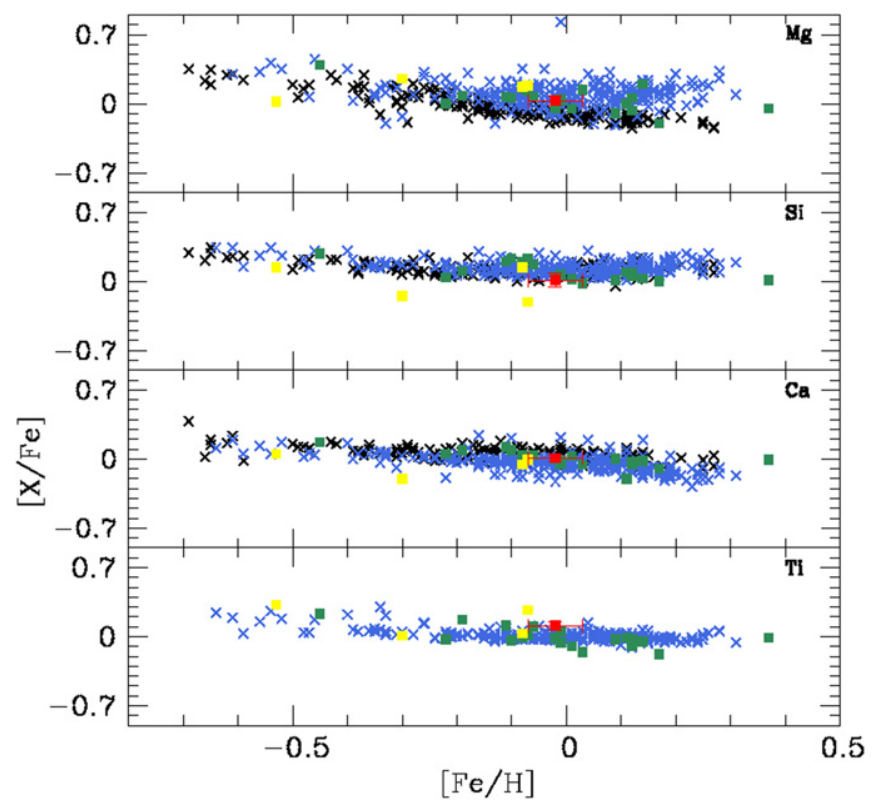

Figure 12. Abundance ratios $[\mathrm{X} / \mathrm{Fe}]$ vs. $[\mathrm{Fe} / \mathrm{H}]$. Symbols have the same meaning as in Figure 11.

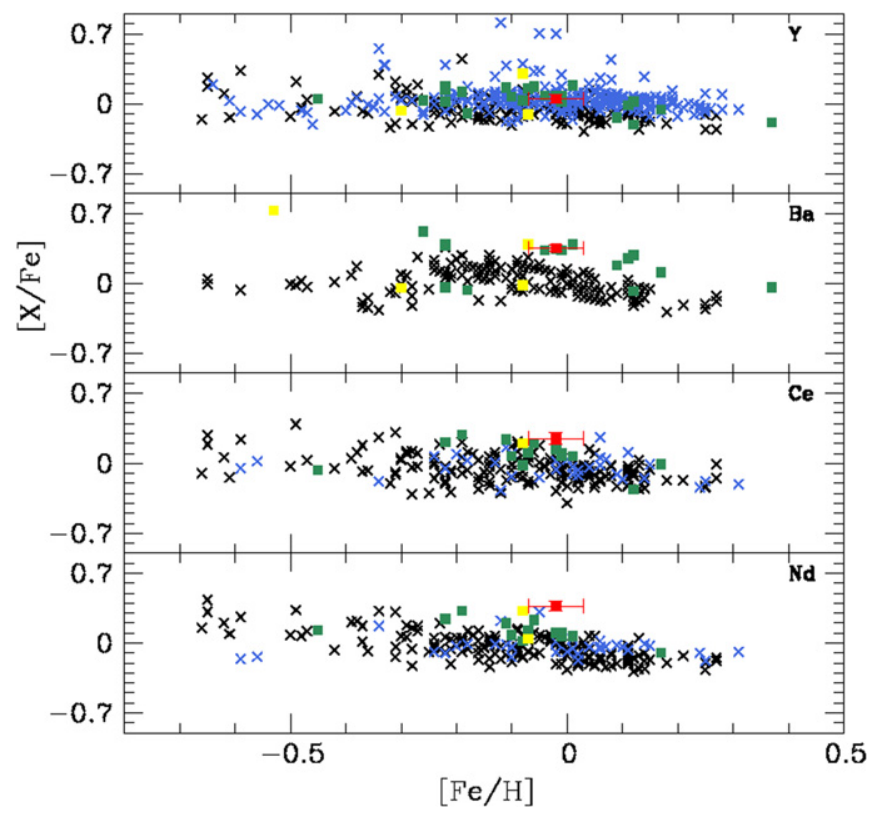

Figure 13. Abundance ratios $[\mathrm{X} / \mathrm{Fe}]$ vs. $[\mathrm{Fe} / \mathrm{H}]$. Symbols have the same meanings as in Figure 11. In the $\mathrm{Y}$ and $\mathrm{Ba}$ panel, we added the results of Mishenina et al. (2013) of the open clusters Berkeley 25, Berkeley 73, Berkeley 75, Ruprecht 4, Ruprecht 7, NGC 6192, NGC 6404, and NGC 6583 (green squares). One field star of our sample (806) exhibits Ba enrichment.

Finally, the overabundance of barium in Cepheid XZ CMa can be explained by an enhancement of $s$-process elements in the interstellar medium (ISM) that produced XZ CMa or by mass transfer in a multiple-star system. Yong et al. (2006) found an enhancement of La for a Cepheid sample in the outer disk and suggested that AGB stars have contributed to the chemical evolution of the outer Galactic disk. XZ CMa is situated at the beginning of the outer disk $\left(R_{\mathrm{GC}}=13 \mathrm{Kpc}\right.$, Yong et al. 2006), which makes XZ CMa one of the cepheid candidates rich in s-process elements formed by this ISM, as suggested by Yong et al. (2006). 


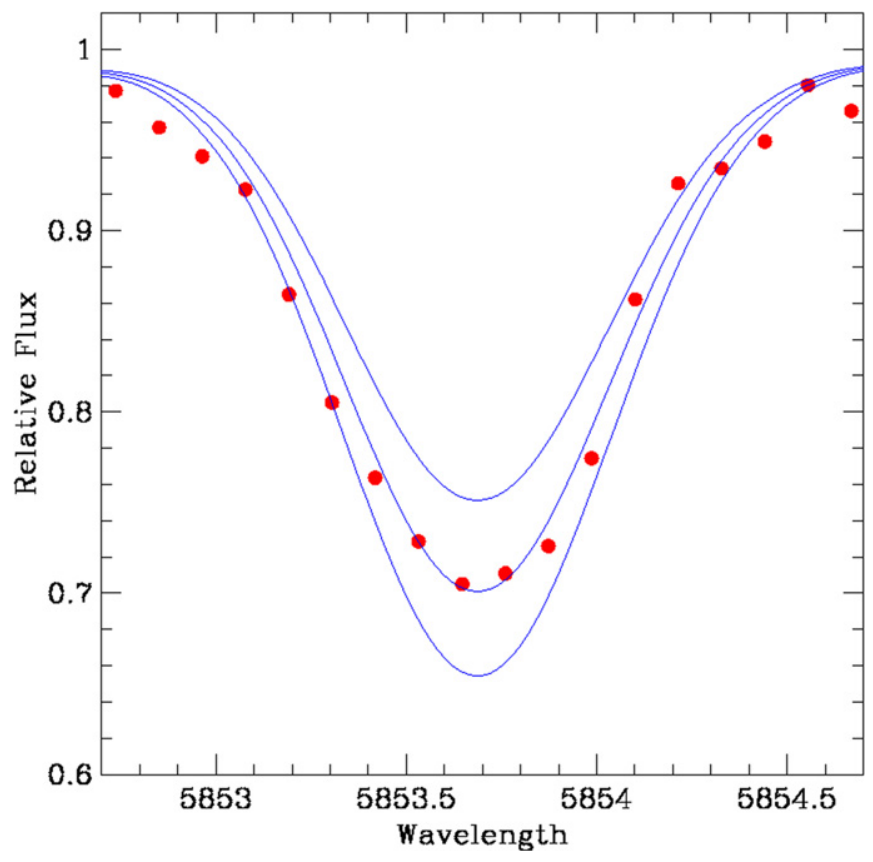

Figure 14. Observed (dotted red line) and synthetic spectra (solid blue lines) in the region of the $\mathrm{Ba}$ II line at $5853 \AA$ for the field giant star 806 . The synthetic spectra were calculated with the barium ratio abundances of $[\mathrm{Ba} / \mathrm{Fe}]=0.33$, 0.73 , and 1.13 .

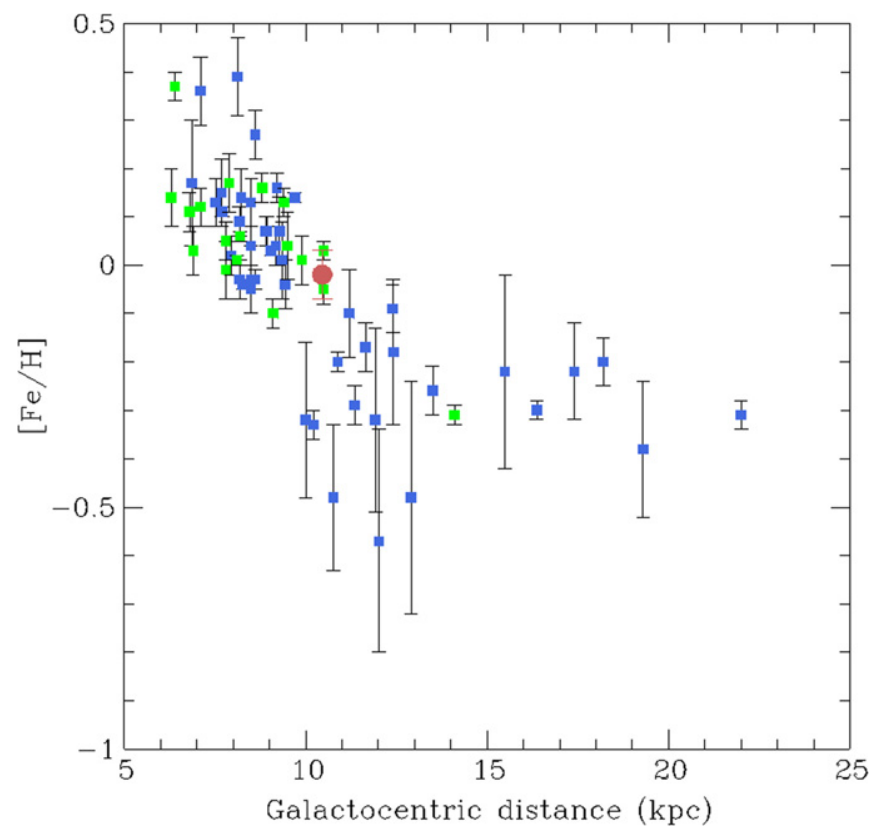

Figure 15. Radial metallicity gradient from Magrini et al. (2009, blue squares) with the addition of Tombaugh 1 (red circle) and additional open clusters analyzed with high-resolution spectroscopy (green squares). See text for details. $R_{\mathrm{GC} \odot}=8.5 \mathrm{kpc}$.

In a binary system, like $\mathrm{Ba}$ and $\mathrm{CH}$ stars, the enrichment of $\mathrm{Ba}$ is a consequence of mass transfer through stellar winds or through Roche-lobe overflow from an AGB star (now the white dwarf) to a less-evolved companion. Turner (1983) suggested the presence of an unresolved blue companion B star to the Cepheid XZ CMa. However, the enrichment of Ba indicates a white dwarf companion to XZ CMa. Thus, we suggest that XZ CMa can belong to a binary system with a white dwarf or a triple system comprising a white dwarf and a B star. About one-third of Galactic Cepheids are known to have companions, and about $44 \%$ of those have more than one companion (Evans et al. 2005). Recently, in the study of the occurrence of classic Cepheids in binary systems, Neilson et al. (2015) pointed out that a fraction of binary systems may evolve to a system composed of a Cepheid with a white dwarf companion. Harris \& Welch (1989) commented that, due to the occurrence of mass transfer in binary Cepheids, an evolutionary connection between $\mathrm{Ba}$ stars and binary Cepheids would be possible. In addition, Gonzalez \& Wallerstein (1996) found significant similarities between binary Cepheids and $\mathrm{Ba}$ and $\mathrm{CH}$ stars, such as orbital parameters and mass range.

Ultraviolet observations of XZ CMa can be used to confirm its binarity and reveal the nature of its companion (e.g., Evans 1992). In the case of a hot companion to XZ CMa, like the B main-sequence star suggested by Turner (1983), the presence of a strong Balmer line, $\mathrm{H} \epsilon(3970.07 \AA)$, in the Cepheid spectrum also can be interpreted as the signature of this blue companion (Kovtyukh et al. 2015). Because of the wavelength coverage of our XZ CMa spectrum (4200-9000 ̊), it was not possible to perform this investigation. The discovery of binary Cepheids is important because unresolved companions are one of the factors that contribute to the scatter around the ridge-line period-luminosity relationship (Szabados \& Klagyivik 2012). In particular, the detection of a Cepheidwhite-dwarf binary will give an important constraint on the most massive progenitors of white dwarfs (Landsman et al. 1996).

Extensive use was made of the WEBDA database maintained by E. Paunzen at the University of Vienna, Austria (http://www.univie.ac.at/webda). The filters used in the program were obtained by B.J.A.T. and B.A.T. through NSF grant AST-0321247 to the University of Kansas. NSF support for this project was provided to B.J.A.T and B.A.T through NSF grant AST-1211621. J.V.S.S. acknowledges the support provided by $\mathrm{CNPq} /$ Brazil Science without Borders program (project No. 249122/2013-8). C.M.B. acknowledges support by the Fondo Nacional de Investigación Científica y Tecnológica (Fondecyt), project No. 1150060. E.C. acknowledges support by the Fondo Nacional de Investigación Científica y Tecnológica (projecto No. 1110100, Fondecyt) and the Chilean Centro de Excelencia en Astrofísica y Tecnologías Afines (PFB 06).

Facilities: Magellan: Baade (IMACS), CTIO:1.0 m (Y4KCam).

\section{REFERENCES}

Ahumada, J. E., \& Lapasset, E. 1995, A\&A, 109, 375

Allende Prieto, C., García López, R. J., Lambert, D. L., \& Gustafsson, B. 1999, ApJ, 527, 879

Alonso, A., Arribas, S., \& Martínez-Roger, C. 1999, A\&AS, 140, 261

Andrievsky, S. M., Lépine, J. R. D., Korotin, S. A., et al. 2013, MNRAS, 428,3252

Andrievsky, S. M., Luck, R. E., \& Korotin, S. A. 2014, MNRAS, 437, 2106 Anthony-Twarog, B. J., Deliyannis, C. P., \& Twarog, B. A. 2014, AJ, 148, 151 Anthony-Twarog, B. J., Deliyannis, C. P., Twarog, B. A., Croxall, K. W., \& Cummings, J. D. 2009, AJ, 138, 1171

Asplund, M., Grevesse, N., Sauval, A. J., \& Scott, P. 2009, ARA\&A, 47, 481 Bellazzini, M., Ibata, R., Monaco, L., et al. 2004, MNRAS, 354, 1263

Bensby, T., Feltzing, S., Lundström, I., \& Ilyin, I. 2005, A\&A, 433, 185

Bertolli, M. G., Herwig, F., Pignatari, M., \& Kawano, T. 2013, arXiv: 1310.4578

Bessell, M. S., Castelli, F., \& Plez, B. 1998, A\&A, 333, 231 
Blackwell, D. E., Booth, A. J., Menon, S. L. R., \& Petford, A. D. 1986, MNRAS, 220, 289

Busso, M., Gallino, R., Lambert, D. L., Travaglio, C., \& Smith, V. V. 2001, ApJ, 557, 802

Busso, M., Gallino, R., \& Wasserburg, G. J. 1999, ARA\&A, 37, 239

Busso, M., Wasserburg, G. J., Nollett, K. M., \& Calandra, A. 2007, ApJ, 671,802

Caldwell, J. A. R., \& Coulson, I. M. 1987, AJ, 93, 1090

Carraro, G., Anthony-Twarog, B. J., Costa, E., Jones, B., \& Twarog, B. A. 2011, AJ, 142, 127

Carraro, G., de Silva, G., Monaco, L., Milone, A. P., \& Mateluna, R. 2014a, A\&A, 566, 39

Carraro, G., Moitinho, A., \& Vźquez, R. A. 2008, MNRAS, 385, 1597

Carraro, G., Monaco, L., \& Villanova, S. 2014b, A\&A, 568, A86

Carraro, G., \& Patat, F. 1995, MNRAS, 276, 563

Carraro, G., Villanova, S., Monaco, L., et al. 2014c, A\&A, 562, 39

Carretta, E., Bragaglia, A., \& Gratton, R. G. 2007, A\&A, 473, 129

Castro, S., Rich, R. M., Grenon, M., Barbuy, B., \& McCarthy, J. K. 1997, AJ, 114,376

Charbonnel, C., \& Lagarde, N. 2010, A\&A, 522, A10

Cowan, J. J., \& Rose, W. K. 1977, ApJ, 212, 149

Crawford, D. L. 1975, AJ, 80, 955

Crawford, D. L. 1979, AJ, 84, 1858

Dardelet, L., Ritter, C., Prado, P., et al. 2015, arXiv:1505.05500

Denisenkov, P. A., \& Denisenkova, S. N. 1990, SvAL, 16, 275

Depagne, E., Hill, V., Spite, M., et al. 2002, A\&A, 390, 187

De Silva, G. M., Sneden, C., Paulson, D. B., et al. 2006, AJ, 131, 455

Dias, W. S., Alessi, B. S., Moitinho, A., \& Lépine, J. R. D. 2002, A\&A, 389,871

Diethelm, R. 1990, A\&A, 239, 186

D’Orazi, V., Biazzo, K., Desidera, S., et al. 2012, MNRAS, 423, 2789

D'Orazi, V., Magrini, L., Randich, S., et al. 2009, ApJL, 693, L31

Drake, J. J., \& Smith, G. 1991, MNRAS, 250, 89

Dressler, A., Hare, T., Bigelow, B. C., \& Osip, D. J. 2006, Proc. SPIE, 6269, $62690 \mathrm{~F}$

Edvardsson, B., Andersen, J., Gustafsson, B., et al. 1993, A\&A, 275, 101

Evans, N. R. 1992, ApJ, 389, 657

Evans, N. R., Carpenter, K. G., Robinson, R., Kienzle, F., \& Dekas, A. E. 2005, AJ, 130, 789

Frebel, A., Casey, A. R., Jacobson, H. R., \& Yu, Q. 2013, ApJ, 769, 57

Gonzalez, G., \& Wallerstein, G. 1996, MNRAS, 280, 515

Gratton, R. 2000, in ASP Conf. Ser. 198, Stellar Clusters and Associations: Convection, Rotation, and Dynamos, ed. R. Pallavicini, G. Micela, \& S. Sciortino (San Francisco, CA: ASP), 225

Gratton, R. G., Carretta, E., Eriksson, K., \& Gustafsson, B. 1999, A\&A, 350,955

Gratton, R. G., \& Sneden, C. 1988, A\&A, 204, 193

Grevesse, N., \& Sauval, A. J. 1998, SSRv, 85, 161

Haffner, H. 1957, ZAp, 43, 89

Harris, H. C., \& Welch, D. L. 1989, AJ, 98, 981

Hauck, B., \& Mermilliod, M. 1998, A\&AS, 129, 431

Herwig, F., Langer, N., \& Lugaro, M. 2003, ApJ, 593, 1056

Iwamoto, K., Brachwitz, F., Nomoto, K., et al. 1999, ApJS, 125, 439

Jacobson, H. R., \& Friel, E. D. 2013, AJ, 145, 107

Jacobson, H. R., Friel, E. D., \& Pilachowski, C. A. 2011, BAAS, 43, 15239

Keeping, E. S. 1962, Introduction to Statistical Inference (London: Van Nostrand)

Kharchenko, N. V., Piskunov, A. E., Schilbach, E., Rser, S., \& Scholz, R.-D. 2013, A\&A, 558, 53

Korotin, S. A., Andrievsky, S. M., Luck, R. E., et al. 2014, MNRAS, 444, 3301

Kovtyukh, V., Szabados, L., Chekhonadskikh, F., Lemasle, B., \& Belik, S. 2015, MNRAS, 448, 3567

Kurucz, R. L. 1993, CD-ROM 13, Atlas9 Stellar Atmosphere Programs and 2 $\mathrm{km} / \mathrm{s}$ Grid (Cambridge, MA: Smithsonian Astrophys. Obs)

Lambert, D. L., Heath, J. E., Lemke, M., \& Drake, J. 1996, ApJS, 103, 183

Landsman, W., Simon, T., \& Bergeron, P. 1996, PASP, 108, 250

Lépine, J. R. D., Cruz, P., Scarano, S., Jr., et al. 2011, MNRAS, 417, 698

Lind, K., Asplund, M., Barklem, P. S., \& Belyaev, A. K. 2011, A\&A, 528, A103

Luck, R. E., \& Bond, H. E. 1981, ApJ, 244, 919

Luck, R. E., \& Bond, H. E. 1991, ApJS, 77, 515

Luck, R. E., \& Heiter, U. 2007, AJ, 133, 2464
Lugaro, M., Herwig, F., Lattanzio, J. C., Gallino, R., \& Straniero, O. 2003, ApJ, 586, 1305

Magrini, L., Randich, S., Romano, D., et al. 2014, A\&A, 563, 44

Magrini, L., Randich, S., Zoccali, M., et al. 2010, A\&A, 523, 11

Magrini, L., Sestito, P., Randich, S., \& Galli, D. 2009, A\&A, 494, 95

Maiorca, E., Randich, S., Busso, M., Magrini, L., \& Palmerini, S. 2011, ApJ, 736, 120

Majaess, D., Carraro, G., Moni Bidin, C., et al. 2013a, A\&A, 560, A22

Majaess, D., Sturch, L., Moni Bidin, C., et al. 2013b, Ap\&SS, 347, 61

Martin, W. C., Fuhr, J. R., Kelleher, D. E., et al. 2002, NIST Atomic Spectra Database (Version 2.0; Gaithersburg, MD: NIST)

McWilliam, A., \& Rich, R. M. 1994, ApJS, 91, 749

Mishenina, T., Korotin, S., Carraro, G., Kovtyukh, V. V., \& Yegorova, I. A. 2013, MNRAS, 433, 1436

Mishenina, T., Pignatari, M., Carraro, G., et al. 2015, MNRAS, 446, 3651

Mishenina, T. V., Bienaymé, O., Gorbaneva, T. I., et al. 2006, A\&A, 456, 1109

Moni Bidin, C., Carraro, G., \& Méndez, R. A. 2012, ApJ, 747, 101

Monroe, T. R., \& Pilachowski, C. A. 2010, AJ, 140, 2109

Neilson, H. R., Schneider, F. R. N., Izzard, R. G., Evans, N. R., \& Langer, N 2015, A\&A, 574, A2

Nissen, P. E. 1988, AJ, 199, 146

Nissen, P. E., Twarog, B. A., \& Crawford, D. L. 1987, AJ, 93, 634

Nordhaus, J., Busso, M., Wasserburg, G. J., Blackman, E. G., \& Palmerini, S. 2008, ApJL, 684, L29

Nucci, M. C., \& Busso, M. 2014, ApJ, 787, 141

Olsen, E. H. 1983, A\&AS, 54, 55

Olsen, E. H. 1988, A\&A, 189, 173

Olsen, E. H. 1993, A\&AS, 102, 89

Olsen, E. H. 1994, A\&AS, 106, 257

Pancino, E., Carrera, R., Rossetti, E., \& Gallart, C. 2010, A\&A, 511, A56

Pavlenko, Y. V., Jenkins, J. S., Jones, H. R. A., Ivanyuk, O., \& Pinfield, D. J. 2012, MNRAS, 422, 542

Pereira, C. B., Sales Silva, J. V., Chavero, C., et al. 2011, A\&A, 533, A51

Piatti, A. E., Claría, J. J., \& Ahumada, A. V. 2004, A\&A, 421, 991

Preston, G. W., \& Sneden, C. 2001, AJ, 122, 1545

Reddy, A. B. S., Giridhar, S., \& Lambert, D. L. 2013, MNRAS, 431, 3338

Reddy, B. E., Bakker, E. J., \& Hrivnak, B. J. 1999, ApJ, 524, 831

Reddy, B. E., Tomkin, J., Lambert, D. L., \& Allende Prieto, C. 2003, MNRAS, 340, 304

Sales Silva, J. V., Peña Suárez, V. J., Katime Santrich, O. J., et al. 2014, AJ, 148,83

Santos, N. C., Lovis, C., Pace, G., Melendez, J., \& Naef, D. 2009, A\&A, 493, 309

Santrich, O. J. K., Pereira, C. B., \& Drake, N. A. 2013, A\&A, 554, A2

Schmidt, E. G. 1984, ApJS, 55, 455

Smiljanic, R. 2012, MNRAS, 422, 1562

Sneden, C. A. 1973, PhD thesis, Univ. Texas

Snowden, M. S. 1975, PASP, 87, 721

Stetson, P. B. 1987, PASP, 99, 191

Szabados, L., \& Klagyivik, P. 2012, Ap\&SS, 341, 99

Tifft, W. G. 1959, ApJ, 129, 241

Tombaugh, C. W. 1938, PASP, 50, 171

Tombaugh, C. W. 1941, PASP, 53, 219

Travaglio, C., Galli, D., Gallino, R., et al. 1999, ApJ, 521, 691

Trippella, O., Busso, M., Maiorca, E., Käppeler, F., \& Palmerini, S. 2014, ApJ, 787, 41

Turner, D. G. 1983, JRASC, 77, 31

Twarog, B. A., \& Anthony-Twarog, B. J. 1995, AJ, 109, 2828

Twarog, B. A., Anthony-Twarog, B. J., Deliyannis, C. P., \& Thomas, D. 2015, AJ, 150,134

Twarog, B. A., Ashman, K., \& Anthony-Twarog, B. A. 1997, AJ, 114, 2556 VandenBerg, D. A., Bergbusch, P. A., \& Dowler, P. D. 2006, ApJS, 162, 375 van Dokkum, P. G. 2001, PASP, 113, 1420

Vásquez, R. A., May, J., Carraro, G., et al. 2008, ApJ, 672, 930

Villanova, S., Randich, S., Geisler, D., Carraro, G., \& Costa, E. 2010, A\&A, 509, A102

Wiese, W. L., Smith, M. W., \& Miles, B. M. 1969, NSRDS-NBS (Washington, D.C.: US Department of Commerce, National Bureau of Standards)

Woosley, S. E., \& Weaver, T. A. 1995, ApJS, 101, 181

Yong, D., Carney, B. W., \& Friel, E. D. 2012, AJ, 144, 95

Yong, D., Carney, B. W., Teixera de Almeida, M. L., \& Pohl, B. L. 2006, AJ, 131,2256 